\title{
Relativistic Quantum Mechanical Condition for Expansion of the Universe
}

\author{
Nishant Kumar Sharma \\ S.B. SGOVT.P, G College, Kumaun University, Nainital, India \\ Email: nkphysics11@gmail.com
}

How to cite this paper: Sharma, N.K. (2021) Relativistic Quantum Mechanical Condition for Expansion of the Universe. Journal of High Energy Physics, Gravitation and Cosmology, 7, 1049-1088. https://doi.org/10.4236/jhepgc.2021.73063

Received: October 23, 2020

Accepted: July 4, 2021

Published: July 7, 2021

Copyright $\odot 2021$ by author(s) and Scientific Research Publishing Inc. This work is licensed under the Creative Commons Attribution International License (CC BY 4.0).

http://creativecommons.org/licenses/by/4.0/

\begin{abstract}
In this manuscript, we will discuss about the quantum mechanical system for the movement of non-intractable particle, non-intractable particle which attends every mass state in the universe, the form of a non-intractable particle is $n=-m$, this manuscript defines the stable cross system for the movement of $n-i$ particles and elementary particles with a perfect black body at centre with proofs of picture of super massive black hole, the linear hamiltonian of the cross quantum mechanical system and with this, it's co-related matrixes, then by the use of cross system of Non-Intractable Particles defining a new right angel theorem. Then the new black body relation free from plank constant depends on non interactive mechanics and $m$, which has already mentioned in non-interactive mechanics and it's relation with galaxies. The unique property of cross system is that it is surrounded by the energy of $10 \mathrm{e}+\mathrm{e}$ always, and at last the relation between zero point energy and dark energy.
\end{abstract}

\section{Keywords}

Riemannian Geometry, Euclidean Geometry, Right Angel Theorem, Factors for the Expansion of Universe, Black Body Radiation, Cosmic Form of the Nishant Effect

\section{Introduction}

We have many theoretical and experimental observations to study how and why universe expands, from Ancient civilizations to sir Edwin Hubble's observation of universe by Hubble telescope, we find that our current universe is expanding, but still some questions arise in every physicist and common man's mind, if 
universe is expanding, then from where the new mass comes and where this mass finds his placed to expand in universe.

So here the point is that first we have to find what is the basic structure of mass, this stage is defined by the $E=1 / 2 h \mathrm{v}$, this is the zero point energy stage of field quanta. The new mass finds that it exists beyond the matter interaction field.

The case of dark matter also occurs as known matter is very less in universe which is interactive with other material bodies while the NON INTERACTIVE matter is very much in universe. The turning of dark matter into normal matter is mentioned in the manuscript. The case of new objects is also shown in the manuscript such as free matter.

There are so many theories and relation in physics where elementary particles change their positions such as Dirac Hole theories. So we study the quantum mechanical condition for the movement of elementary particles. For this, we use relativistic quantum mechanics as a tool to understand quantum mechanical and relativistic condition for the exchange of elementary particles. Here we will study the relativistic quantum mechanical energy, momentum relation to understand the expansion of the universe and the factors for the expansion of universe. The relativistic quantum-mechanical condition is stated as

$$
\frac{i \hbar \partial \psi}{d t}= \pm \sqrt{(P c)^{2}}+\left(m c^{2}\right)^{2}
$$

Now here we try to interpret the wave structure and properties presented by this equation by considering each side and its relative terms. Let us consider first L.H.S.

\section{Equations and String Structures of Quantum Wave Function}

\section{L.H.S $(i \hbar \partial \psi / d t)$ :}

A. The Q. Mech. Wave $\psi$. As many theories and experiments predicted vary qualities of waves with different structures such as circular, spherical wave, but the most common structure for a wave considered as spherical, the reason behind choosing the spherical shape as many atomic model theories successfully predicted the spherical shape of atomic orbital's which was confirmed by experimental proofs, except their an exception for hydrogen atom which presents a circular wave. So for a normal condition the representative wave taken as spherical, "as shown in Figure 1".

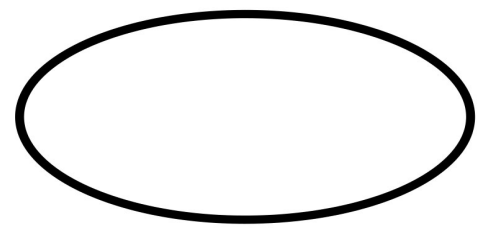

Figure 1. $\psi$ presentation of a wave. 
B. Now considering the differentiable part is $(\partial / d t)$, now this represents two parts:

1) The wave is differentiating w.r.t or moving under time $t$.

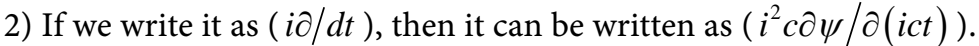

So by the first view the first diffr. W.r.t to time $(t)$, looks as the fraction of above presented wave "as shown in Figure 2".

As if look the wave then and study its geometry than for space and time, it represents the Riemannian geometry with the equation Euclidean geometry $\left(d S^{2}=d X u d X v\right.$ guv $)$.

Now as predicted by Einstein the curve geometry turned into straight geometry in special condition or the case of Riemannian geometry turned into Euclidean geometry (Euclidean geometry $\left(d S^{2}=d x 1^{2}+d x 2^{2}+d x 3^{2}+d x 4^{2}\right.$ here $(X 4=i c t)$.

In normal conditions, the curve wave of Riemannian geometry looks as straight line wave of Euclidean geometry (Figure 3).

Here the $\hbar$ represents the minimum required energy per unit time for wave which is $\hbar=4.2203 * 10^{-34} \mathrm{~J} \cdot \mathrm{s}$.

So the wave equation has the form of ( $c \hbar \partial \psi / \partial i c t)$.

Actual geometry seems as Figure 4.

The remaining term representing the movement of given wave with respect to absolute velocity $C$ speed of light.

Now looking at the right hand side of Equation (1).

R.H.S

$\pm \sqrt{(P c)^{2}}+\left(m c^{2}\right)^{2} \psi$ the part deal with the energy distribution in above shown wave.

Now if we assume that this shows the wave similar as L.H.S than let's just see the distribution of energy in the above wave

$$
\begin{aligned}
& \pm \sqrt{m^{2} c^{4}}+(P c)^{2} \cdot \psi, \text { now using binomial theorem } \\
& \pm \sqrt{m^{2} c^{4}}+(P c)^{2} \cdot \psi= \pm\left(1+\frac{1}{2} !\left(\left(m c^{2}\right)^{2}\right)+(P c)^{2}\right)
\end{aligned}
$$

(Here neglecting higher power term)

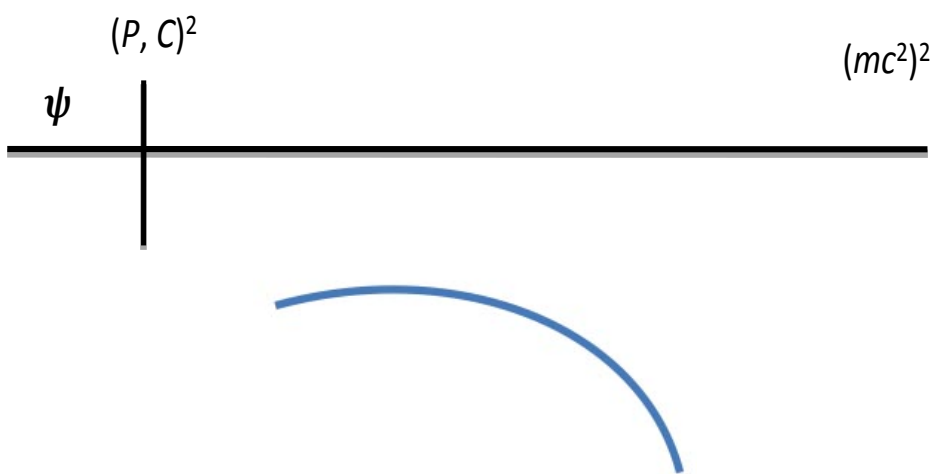

Figure 2. fraction of the quantum wave $\mathrm{w}, \mathrm{r}, \mathrm{t}$ to time $t(\partial \psi / d t)$. 


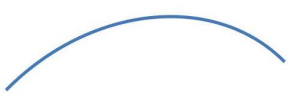

Figure 3. Riemannian geometry ( $d S^{2}=d X u d X v$ guv ) Euclidean geometry $\left(d S^{2}=d x 1^{2}+d x 2^{2}+d x 3^{2}+d x 4^{2}\right)$.

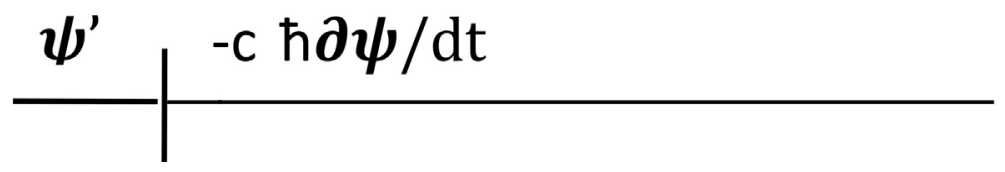

Figure 4. The linear expansion in relativistic quantum mechanical conditions.

so the Equation (1) looks as: $i \hbar \partial \psi / d t= \pm\left(1+\frac{1}{2} !\left(\left(m c^{2}\right)^{2}\right)+(P c)^{2}\right) \psi$.

The form of sign distribution is ignored till final Figure 4 arrives.

1) $\left(m c^{2}\right)^{2}=\left(m c^{2}\right)\left(m^{\prime} c\right)^{2}$ Where $m$ and $m$ ' vary as their velocity with respect to speed of Light $C$.

2) $(P C)^{2}=(P c)\left(P^{\prime} C\right)$.

Now if substituting $P=-i \hbar \partial \psi / d x$ and distributing "signs" on Figure 5. Then Figure 5 shown above justify the sign balancing of upper quantum state and lower classical state and as we can see that shown Figure 6 justify that both classical energy state and quantum energy state is bound in the same Figure 7 and there mass vary relatively (as $m, m^{\prime}$ ) so the case of classical mechanics and quantum mechanics are in same Figure 8, Figure 9 and their mass vary relatively with a perfect BLACK BODY AT CENTER which justifies the condition.

Here the travel from condition $(\psi, a, d)$ to attained the mass holding states is non-interactive mechanics and the particles which poss the mechanics called non-interactive particle in Figure 10.

\section{Linear Hamiltonian, System Stability and Equation}

In this section we will check the stability of the above mentioned system for the interchange of the elementary. For this we check the stability of the linear Hamiltonian of the above mentioned system.

According to above figure linear Hamiltonian is:

$$
\begin{gathered}
H=+\left(-i \hbar \frac{\partial \psi}{d x}\right) \cdot C-m(C)^{2} \\
H=P \cdot c-m(C)^{2}
\end{gathered}
$$

using constant $\alpha$ and $\beta$ in above equation

$$
\begin{gathered}
H=\alpha \cdot(P \cdot c)-\beta \cdot\left(m(C)^{2}\right) \\
i \hbar \partial \psi / d t=\alpha \cdot(P \cdot c)-\beta \cdot\left(m(C)^{2}\right)
\end{gathered}
$$




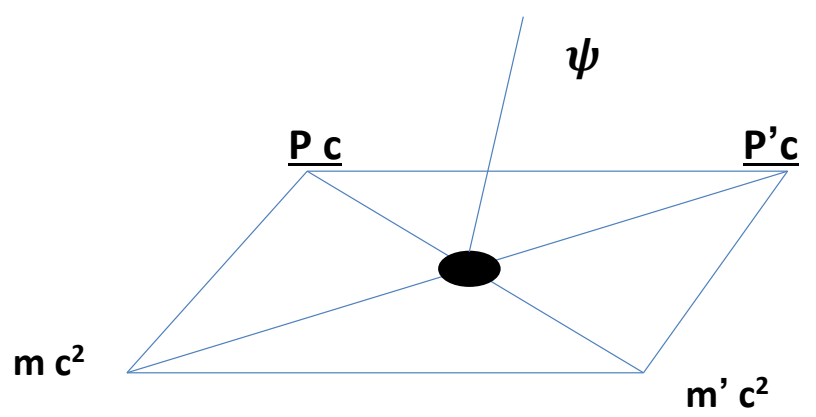

Figure 5. Relativistic quantum mechanical for the movement of non intractable particles.

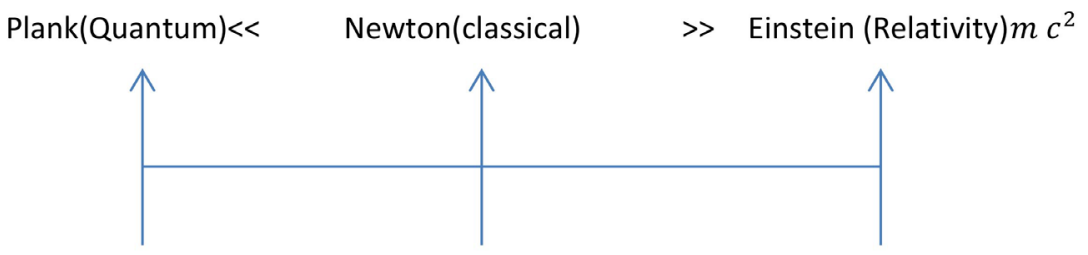

Figure 6. Both classical energy state and quantum energy state.

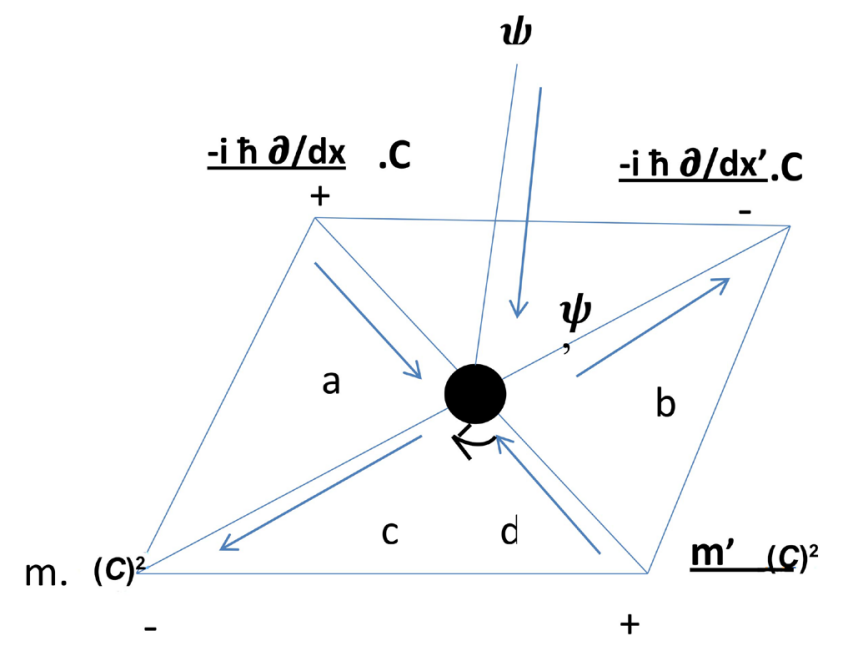

Figure 7. Mass varies relatively (as $m, m^{\prime}$ ).

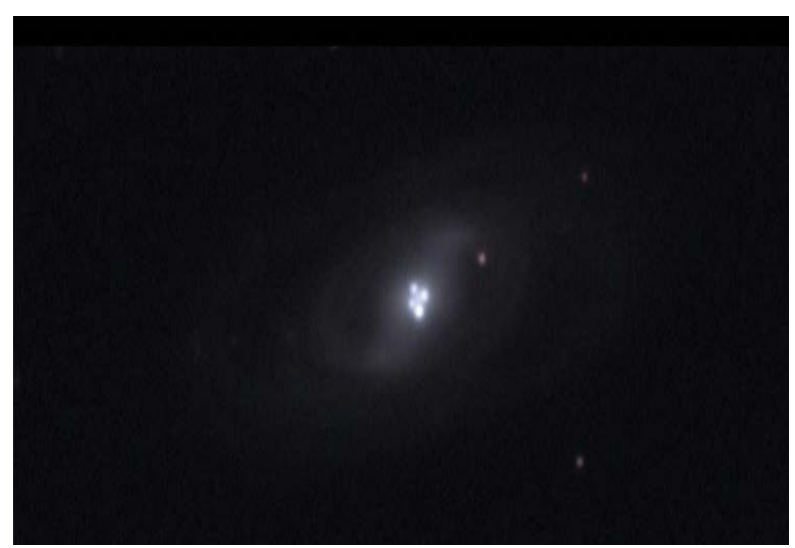

Figure 8. Mass varies relatively with a perfect BLACK BODY AT CENTER. Source: Hubble space telescope. 


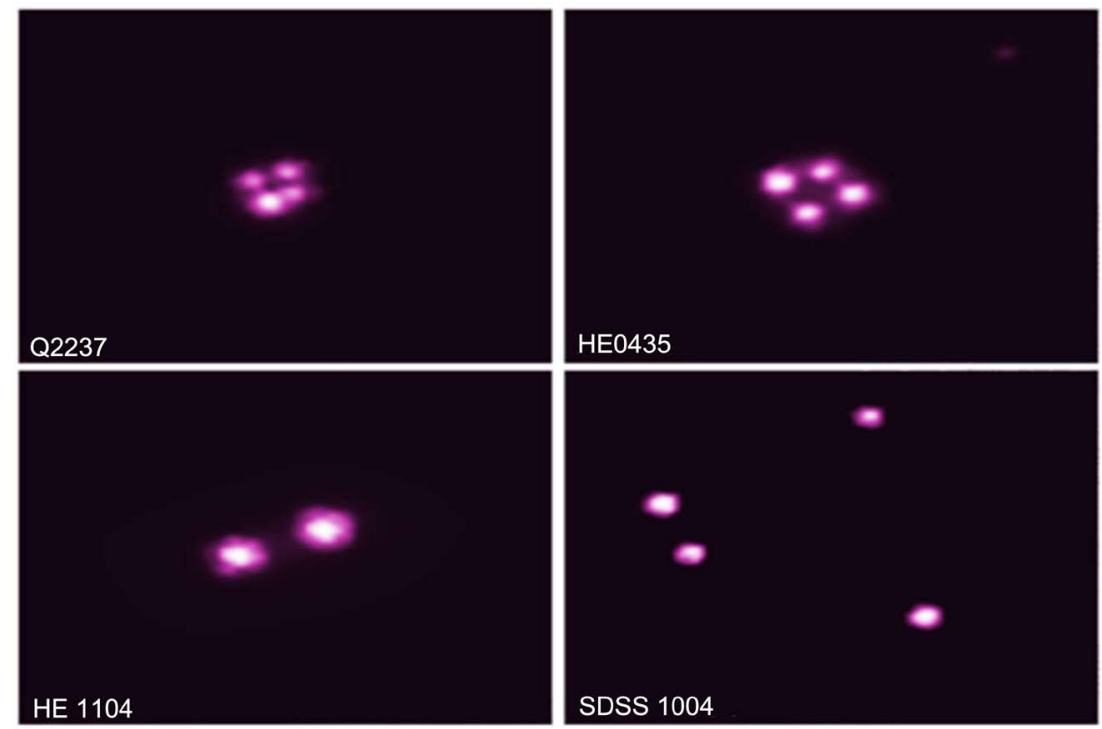

The lensed quasars in this study. NASA/CXC/Univ. of Oklahoma/X. Dai et al.

Figure 9. Mass varies relatively with a perfect BLACK BODY AT CENTER. Source: Hubble space telescope.

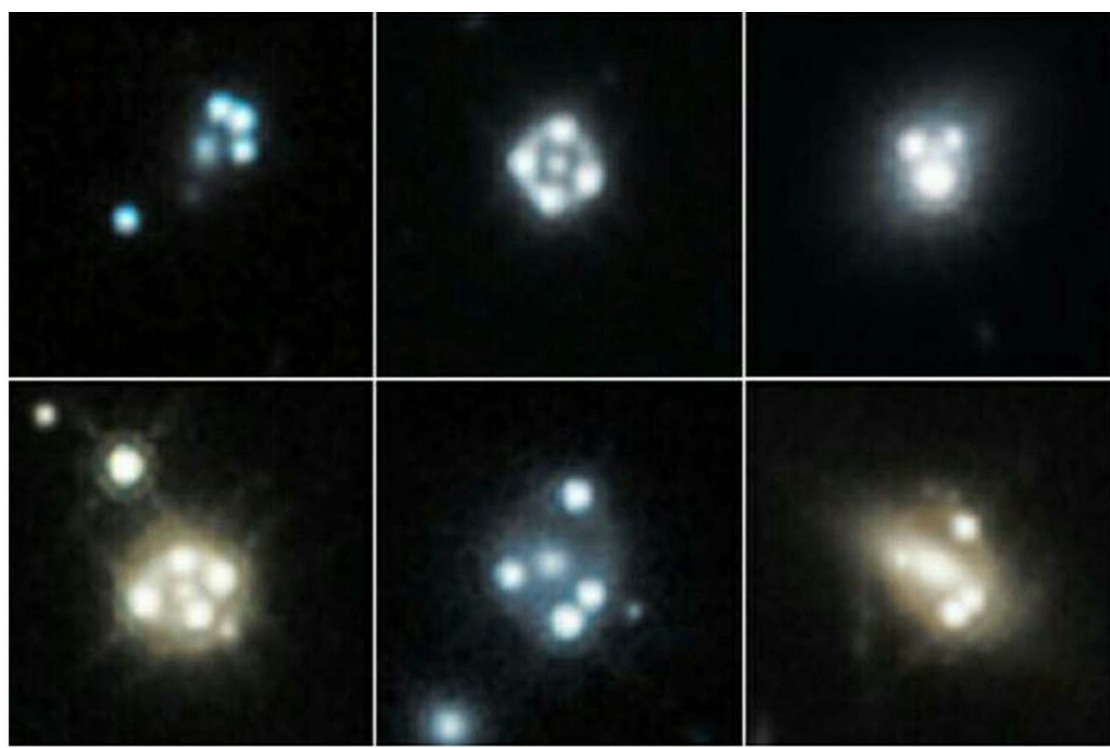

\section{Each of these HUbble Space Telescope snapshots r...}

Figure 10. Non-interactive particle.

$$
\begin{gathered}
i \hbar \partial \psi / d t-\alpha \cdot(P \cdot c)+\beta \cdot\left(m(C)^{2}\right)=0 \\
\left(E-\alpha \cdot(P \cdot C)+\beta \cdot\left(m(C)^{2}\right)\right) \psi=0
\end{gathered}
$$

Multiplying by $E+\alpha \cdot(P \cdot C)-\beta \cdot\left(m(C)^{2}\right)$

$$
\left(E-\alpha \cdot(P \cdot c)+\beta \cdot\left(m(C)^{2}\right)\right) \cdot\left(E+\alpha \cdot(P \cdot c)-\beta \cdot\left(m(C)^{2}\right)\right)=0
$$




$$
\begin{gathered}
E^{2}+E \cdot \alpha \cdot(P \cdot C)-E \beta \cdot\left(m(C)^{2}\right)-\alpha \cdot(P \cdot C) \cdot E-\alpha^{2} \cdot P^{2} \cdot(C)^{2} \\
+\alpha \cdot(P \cdot C) \cdot \beta \cdot\left(m(C)^{2}\right)+E \cdot \beta \cdot\left(m(C)^{2}\right) \\
+\beta \cdot\left(m(C)^{2}\right) \cdot \alpha \cdot(P \cdot C)-\beta^{2} \cdot m^{2} \cdot(C)^{4}=0 \\
E^{2}+2 m^{2} \cdot(C)^{4} \alpha \cdot \beta-\beta^{2} \cdot m^{2} \cdot(C)^{4}=0
\end{gathered}
$$

As $i \cdot \alpha \cdot \beta=0$

$$
\begin{gathered}
i \cdot \beta^{2}=1 \\
E^{2}-m^{2} \cdot(C)^{4}=0
\end{gathered}
$$

For $E=m \cdot(C)^{2}$

$$
\begin{gathered}
m^{2} \cdot(C)^{4}-m^{2} \cdot(C)^{4}=0 \\
0=0 \\
\text { L.H.S }=\text { R.H.S }
\end{gathered}
$$

This proofs the above figure linear Hamiltonian is stable.

In matrix form

$$
\begin{aligned}
\left(\begin{array}{ll}
1 & 1 \\
1 & 1
\end{array}\right) & =\left(\begin{array}{ll}
1 & 1 \\
1 & 1
\end{array}\right),\left(\begin{array}{ll}
-1 & -1 \\
-1 & -1
\end{array}\right)=\left(\begin{array}{ll}
1 & 1 \\
1 & 1
\end{array}\right),\left(\begin{array}{ll}
1 & -1 \\
1 & -1
\end{array}\right)=\left(\begin{array}{ll}
1 & 1 \\
1 & 1
\end{array}\right), \\
\left(\begin{array}{ll}
-1 & 1 \\
-1 & 1
\end{array}\right) & =\left(\begin{array}{ll}
1 & 1 \\
1 & 1
\end{array}\right),\left(\begin{array}{cc}
-1 & 1 \\
1 & -1
\end{array}\right)=\left(\begin{array}{cc}
1 & 1 \\
1 & 1
\end{array}\right),\left(\begin{array}{cc}
1 & -1 \\
-1 & 1
\end{array}\right)=\left(\begin{array}{ll}
1 & 1 \\
1 & 1
\end{array}\right), \\
& \left(\begin{array}{cc}
-1 & -1 \\
1 & 1
\end{array}\right)=\left(\begin{array}{ll}
1 & 1 \\
1 & 1
\end{array}\right),\left(\begin{array}{cc}
1 & 1 \\
-1 & -1
\end{array}\right)=\left(\begin{array}{ll}
1 & 1 \\
1 & 1
\end{array}\right)
\end{aligned}
$$

\section{Dirac Hamiltonian and Need of Non Intractable Particle}

Dirac provided the Hamiltonian $H=\alpha \cdot(P \cdot c)+\left(m(C)^{2}\right)[1]$.

According to above mentioned relativistic quantum figure this is a diagonal hamiltonian, not a linear hamiltonian but a rectangular linear Hamiltonian the only possibility for this condition is that in above mentioned relativistic quantum figure is that the relativistic energy exchange in form of charge between the linear relativistic quantities $m(C)^{2}$ and $m^{\prime}(C)^{2}$ the flow of energy will be from $m$ to $m$ ' from -'ve to +'ve and from $+m(C)^{2}$ to $+P . C$ then the Diagonal Hamiltonian of dirac will be formed $H=\alpha \cdot(P \cdot c)+\left(m(C)^{2}\right)$.

\section{The Need and Existence of Non-Intractable Particle}

In the above condition for forming a Dirac Hamiltonian the relativistic energy exchange is required in then the Hamiltonian will be $H=\alpha \cdot(P \cdot c)+\beta \cdot\left(m(C)^{2}-\left(m^{\prime}(C)^{2}\right)\right)$, if $m=m^{\prime}$ then the remaining term will be $H=\alpha \cdot(P \cdot C)$ But practically, this is not possible so the modified mass is $m-m^{\prime}=M$, Generally it is considered as mass defect. But generally if the two solid bodies of same Masses are Striking to each other then the concept of mass defect is not given sufficient answer, here the concept of non intractable particle as mentioned in law of gravitation For non intractable particles is required that in two bodies of same masses are able to Generate minimum 1 Non Intractable 
Particles between them when the distance between. Them is minimum 1 unit and the masses are equal. Similarly in above mentioned relativistic quantum figure minimum one non intractable particle will generate between two relativistic bodies and after the destruction of relativistic masses, the non intractable particle will emit matter according to the property of Non Intractable Particles which is $N=-M$.

According to non interactive mechanics non intractable t only enter in positive charge field so the flow of energy is from -'ve to +'ve and then this energy moves to create the diagonal Hamiltonian so the Hamiltonian is

$H=\alpha \cdot(P \cdot c)+\beta \cdot\left(m(C)^{2}+N \cdot(C)^{2}-\left(m^{\prime}(C)^{2}\right)\right)$ for $m=m^{\prime}$ this condition is $H=\alpha \cdot(P \cdot C)+\beta \cdot N \cdot(C)^{2}$. Here non intractable Particle emits matter and the value is $H=\alpha \cdot(P \cdot C)+\beta \cdot(-M)^{\prime}(C)^{2}$. Here the value of -'ve denotes the disintegration of matter neglecting this we get $H=\alpha \cdot(P \cdot C)+\beta \cdot(M)^{\prime}(C)^{2}$. This is the Required Dirac Hamiltonian. The flow of energy is centralized and expanded by following clockwise rotation as mentioned in figure. The mass of Non intractable particle is $N=13.4160753862 \mathrm{Gev}-\mathrm{sec} / C^{2}$ [2] for $N=-M$ the mass of Non intractable.

Particle is $M=13.4160753862, \mathrm{Gev}-\mathrm{sec} / C^{2}$ at $t=10^{-10}$ see [3] mass value is obtained for light particles $M=13$. Gev-secsec $/ C^{2}$ at $t=10^{-9} \mathrm{sec}$. Both values are according to the paper on the development of photo electric effect.

Currently we can't observe the particle of this energy range so we need new generation of particle accelerator to detect this range of particles. But this type of particles is available naturally so we can form Dirac Hamiltonian conditions in laboratory.

\section{Right Angel Triangle Theorem-Geometrical Aspect of Expansion of Universe}

"IN a right angle triangle the energy of the hypotenuse ( $c d)$, addition of perpendicular $(b c)$ and base $(b d)$ energies and line which is going throw the base and perpendicular lines $(a b)$ energies are equals".

In mathematics and physics Pythagoras theorem is a well-known theorem which describes the relation for right angle triangle, in field of physics it plays an important role in describing the structure of the fabric of space time, by use of relativistic quantum mechanics we have established the system for the movement of non intractable particles and expansion of field and now we use non interactive mechanics to describe the inner structure of the quantum mechanical system and then we describe the right angle triangle theorem, the use of this theorem is to describe the expansion of relativistic quantum field by this theorem describe that expansion of universe is equal (Figure 11).

Now looking the above relativistic quantum Figure 12 and assuming its fourth part.

Suppose we have a right triangle whose angle $\mathrm{dbc}$ is a right angle. It has four lines base "bd" line bc which is perpendicular to bd and hypotenuse "cd". And line $a b$ which is perpendicular to point $b$. 


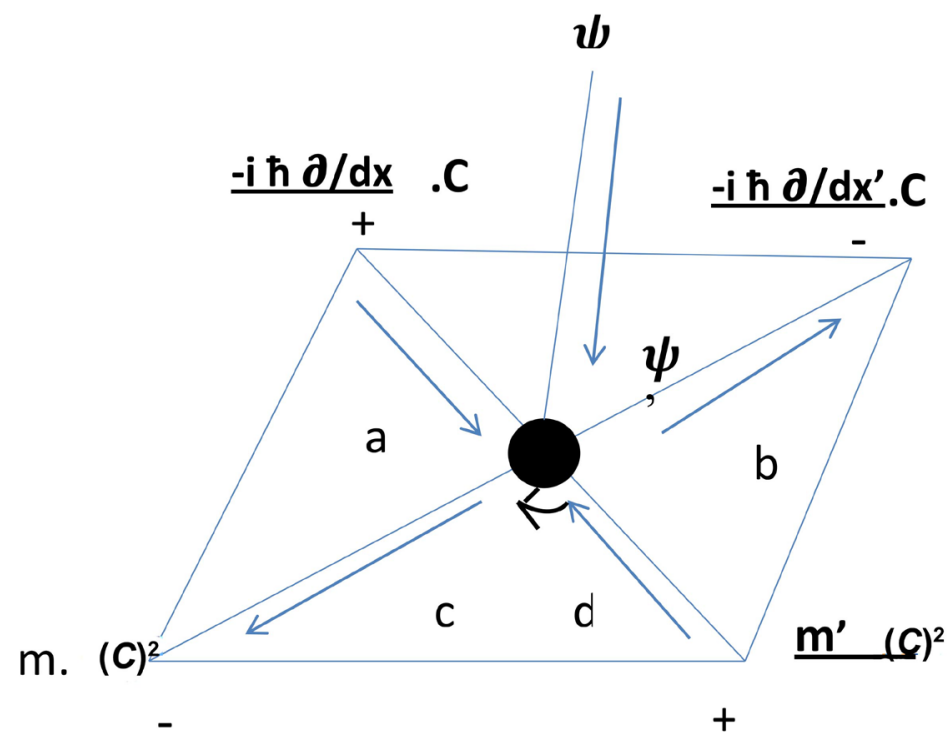

Figure 11. In horizontal and vertical directions.

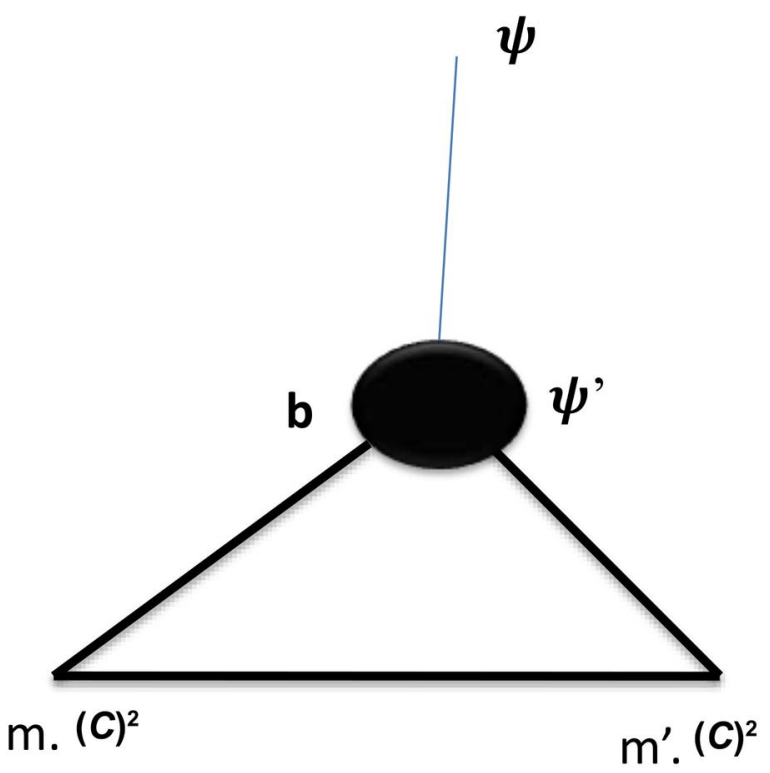

Figure 12. Forth part of Figure 11.

Now the right angle triangle theorem is:

"The value of hypotenuse 'cd' of a right triangle bcd equals to the perpendicular line $\mathrm{ab}$, and the system $\mathrm{bc}+\mathrm{bd}, \mathrm{dc}$ and $\mathrm{ab}$ are equal to each other".

Mathematically-ab $=b c+b d=c d$.

For proving that let's take a look at nishant effect of non-interactive mechanics which gives three eq. of energy and a figure:

1) $E^{n}=(N+M) \cdot\left(g i e^{\alpha+\beta E i}-e^{-\alpha-\beta E i}\right)^{2 \cdot n}[4]$.

2) $E^{n}= \pm \sqrt{N^{2}+2 N M+M^{2}} \cdot\left(g i e^{\alpha+\beta E i}-e^{-\alpha-\beta E i}\right)^{2 \cdot n}$ [4].

3) $E^{n}=\frac{2(h V m a x-h V o)}{v^{2}} \cdot\left(g i e^{\alpha+\beta E i}-e^{-\alpha-\beta E i}\right)^{2 \cdot n}[4]$. 
So the combined forms of these relations are Figure 13.

First the Equation (1) and (2) we have

1) $E^{n}=(N+M) \cdot\left(g i e^{\alpha+\beta E i}-e^{-\alpha-\beta E i}\right)^{2 \cdot n}$, for $N=0, C=g i e^{\alpha+\beta E i}-e^{-\alpha-\beta E i}$ and $n=1$

$$
E=M \cdot(C)^{2}
$$

2) $E^{n}= \pm \sqrt{N^{2}+2 N M+M^{2}} \cdot\left(g i e^{\alpha+\beta E i}-e^{-\alpha-\beta E i}\right)^{2 \cdot n}$, for $N=0$, $C=g i e^{\alpha+\beta E i}-e^{-\alpha-\beta E i}$ and $n=1$ And taking “+Ve" value we have

$$
E=M \cdot(C)^{2}
$$

3) $E^{n}=\frac{2(h V \max -h V o)}{v^{2}} \cdot\left(g i e^{\alpha+\beta E i}-e^{-\alpha-\beta E i}\right)^{2 \cdot n}$ for $h \max \gg h V o$, $C=g i e^{\alpha+\beta E i}-e^{-\alpha-\beta E i}$ and $n=1$

$$
E=\frac{2(h V \max )}{v^{2}} \cdot(C)^{2}
$$

Now for $\frac{(c n-i)^{2}}{v^{2}}=1$ if $c$ is $c n-i$ non intractable particle and $v$ is normal light then difference is $20,817.22 \mathrm{~m} / \mathrm{sec}$ this distance at this velocity level is almost approximation so applying the condition in above equation we have.

$$
E=2 h v m a x
$$

According to theorem

$$
\begin{gathered}
\mathrm{ab}=\mathrm{bc}+\mathrm{bd}=\mathrm{cd} \\
2 h \operatorname{Vmax}=M \cdot(C)^{2}+M \cdot(C)^{2} \\
2 E=2 E \text { almost }
\end{gathered}
$$

And from Figure 12 "cd" $=2 M \cdot(C)^{2}=2 E$.

This proves the theorem

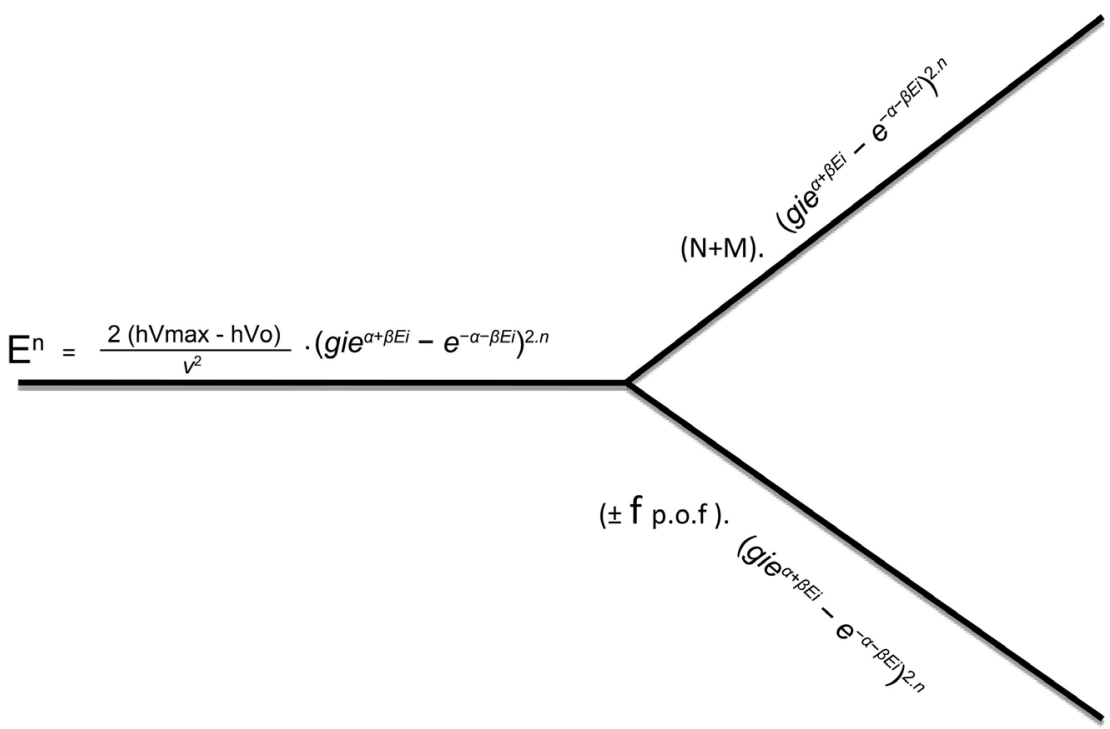

Figure 13. The Nishant effect of Non Interactive mechanics. 


$$
\mathrm{ab}=\mathrm{bc}+\mathrm{bd}=\mathrm{cd}
$$

This states that the expansion of universe is equal in horizontal and vertical same so the universe is expanding as vertically downward and linearly horizontal. So the expansion of universe depends on the vertical and downward pressure of cosmic rays and astroparticles and due to that universe expands horizontally. This is the reason that many structures such as mountains poss this type structure on earth3, mars and other planets.

So if NASA and other space agencies are trying to establish civilization on other planets than they should choose planets with mountains.

The Limit of Expansion of This Relativistic Quantum System

In the above triangle system (Figure 12) as the vertical downward pressure continuously applies on the point $\mathrm{b}$ the sides $\mathrm{cb}$ and bd continuously moves downward as the point comes as Figure 14.

This is the one fourth part of relativistic quantum Figure 15, taking all fourth part for this condition we find that all fourth parts expand the centre body to the whole system and it's expanded Figure 16 seems as follows:

This is the expanding limit of a relativistic quantum system in the universe after achieving this state respective relativistic quantum system becomes stable and new relativistic quantum systems come in place of it and then the whole process repeats itself again.

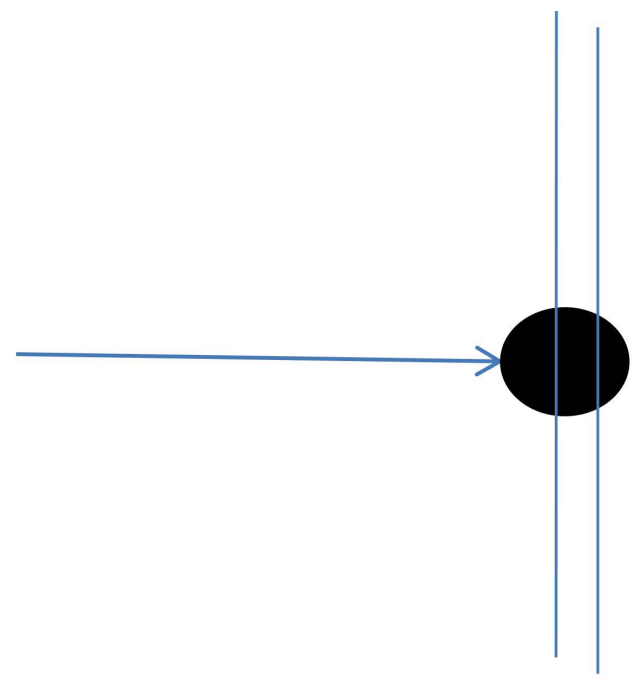

Figure 14. Horizontal and vertical impact on class zero field.

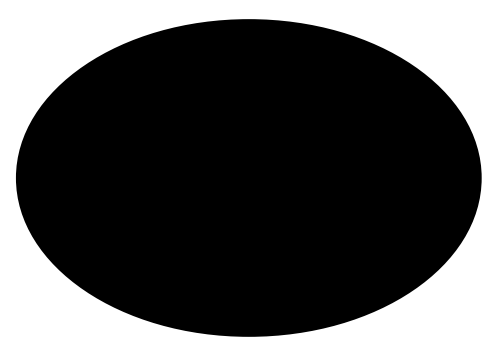

Figure 15. All fourth parts. 


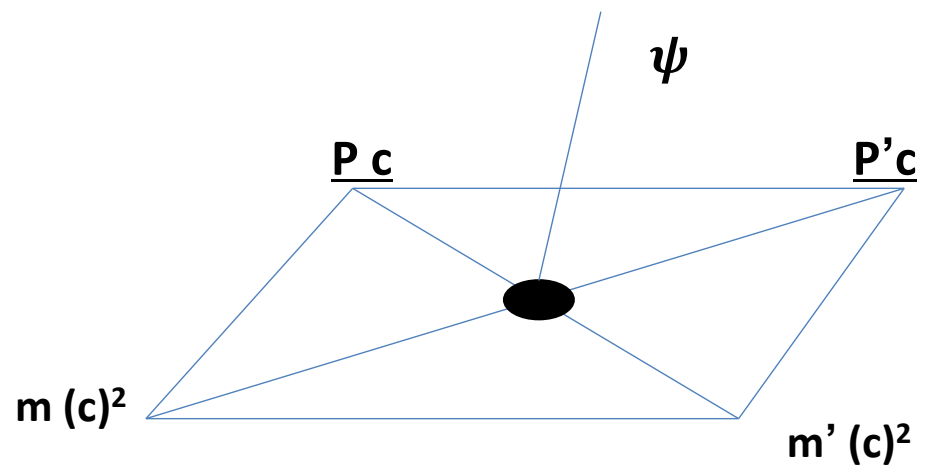

Figure 16. Expanded whole system.

\section{Condition for Continuous Expansion of Universe}

As Expansion of a relativistic quantum system completes the process of Expansion of universe didn't stop practically we find that after the expansion of a galaxy the expansion of universe doesn't stop. The formation of other galaxies also proceeds. Now we will study the conditions we are responsible for the expansion of universe. These are:

1) Free matter.

2) Velocity of non-intractable particle with respect to light and black hole.

3) Particles entering rule differences from normal matter to dark matter.

4) Converted matter into particle.

5) The dark energy need for expansion of universe.

Now we will study each of these cases:

\section{1) Free Matter}

In above described relativistic quantum mechanical figure the there is relativistic mass energy relations are provided by use of these mass energy relations we will describe the existence of matter which is free from work Energy bond of Universe. Suppose two bodies of mass $m$ and $m$ moving with the velocity of light $C$ respect to each other then their energies will be $E=m(C)^{2}$ and $E^{\prime}=m^{\prime} \cdot(C)^{2}$. Due to energy exchange between them, the temperature produces between them are $T=2 M \cdot(C)^{2}$ Here $M=\left(m+m^{\prime}\right)$. Here we can use the relation for free matter during the energy exchange of two space bodies. $M$ free = $2 T /(C)^{2}$.

Checking the accuracy of this equation with $y$ heoretical and experimental results:

a) For Electron

For electron the mass is $m=9.1 \times 10^{-31} \mathrm{~kg}$. The velocity of light is $C=2.99792458 \times 10^{8} \mathrm{~m} / \mathrm{sec}$. Applying this in above equation $T=2 \mathrm{~m} \cdot(C)^{2}$. We have $T=2 \times 9.1 \times 10^{-31} \times 8.987 \times 10^{16}=163.57 \times 10^{-15}=-9.4452$ Kelvin changing it into Celsius: $c=k-273, c=-9.4452-273.15, T=-282.5952$ Celsius.

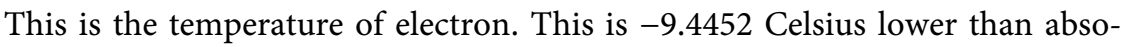
lute zero. It is approximation and satisfaction of rule. But as experiments say we 
need accuracy so we need equal to absolute zero or higher than absolute zero temperature.

b) For Light

The mass of light $m=13.416075386 \mathrm{Gev} \cdot \mathrm{sec} / C^{2}$.

The velocity of light is $C=2.99792458 \times 10^{8} \mathrm{~m} / \mathrm{sec}$.

Applying these in equation $T=2 m(C)^{2}$.

We have

$$
\begin{gathered}
T=2 \times 13.4160753862 \mathrm{Gev} \cdot \mathrm{sec} / C^{2} \times 2.99792458^{2} \times 10^{16} \mathrm{~m}^{2} / \mathrm{sec}^{2} \\
T=34.4781692671 \mathrm{Gev} \cdot \mathrm{m}^{2} / C^{2} \cdot \mathrm{sec}
\end{gathered}
$$

In $\mathrm{kg}$

$$
T=-21.8148510461 \mathrm{~kg} \cdot \mathrm{m}^{2} / \mathrm{sec}
$$

If

$$
1 \mathrm{~kg}=1 \text { Kelvin and for area unit }=1 \mathrm{~m}^{2} \text { and time unit }=1 \mathrm{sec}
$$

Then

$$
T=-21.8148510461 \mathrm{k}
$$

In Celsius

$$
c=k-273.15
$$

For the velocity of light $C=-21.8148510461-273.15$

$$
T=-294.9648510461 \text { Celsius }
$$

This is the temperature of light in Celsius, this means that light photons also have the property of temperature emission. And due to this light photons also extract free matter in universe. Thus light photons are also not the particle which not emits matter. This states that light photons are unstable in the universe.

c) For Non Intractable Particles

The mass of non intractable particles $m=13.4160753862 \mathrm{Gev} \cdot \mathrm{sec} / C^{2} \quad$ [2].

The velocity of light is $C=2.9981327522 \times 10^{8} \mathrm{~m} / \mathrm{sec}$.

Applying these in equation $T=2 m \cdot(C)^{2}$.

We have

$$
\begin{gathered}
T=2 \times 13.4160753862 \mathrm{Gev} \cdot \mathrm{sec} / \mathrm{C}^{2} \times 2.9981327522^{2} \times 10^{16} \mathrm{~m}^{2} / \mathrm{sec}^{2} \\
T=23.2405510503 \mathrm{Gev} \cdot \mathrm{m}^{2} / C^{2} \cdot \mathrm{sec}
\end{gathered}
$$

In $\mathrm{kg}$

$$
T=-23.1789346688 \mathrm{~kg} \cdot \mathrm{m}^{2} / \mathrm{sec}
$$

If $1 \mathrm{~kg}=1$ Kelvin and for area unit $=1 \mathrm{~m}^{2}$ and time unit is $=1 \mathrm{sec}$

Then

$$
T=-23.1789346688 \mathrm{~kg}
$$

In Celsius

$$
c=k-273.15
$$

$C=-23.1789346688-273.15$ 


$$
T=-296.3289346688 \text { Celsius }
$$

This is the temperature of non intractable particle in celsius, this means that non intractable particles also have the property of temperature emission. And due to this non intractable particles are also have matter emission property. This states that non intractable particles are also unstable in universe.

For an object or particle with Maas of $=13.4160753862 \mathrm{Gev}-\mathrm{sec} / \mathrm{C}^{2}$ and Velocity $=212,000,000 \mathrm{~m} / \mathrm{sec}$.

Applying in equation $T=2 m \cdot(C)^{2}$.

We have to add them in equation

$$
\begin{gathered}
T=2 \times 13.4160753862 \mathrm{Gev} \cdot \mathrm{sec} / C^{2} \times 2.12^{2} \times 10^{16} \mathrm{~m}^{2} / \mathrm{sec}^{2} \\
T=20.6202755143 \mathrm{Gev} \cdot \mathrm{m}^{2} / C^{2} \cdot \mathrm{sec}
\end{gathered}
$$

In $\mathrm{kg}$

$$
T=-23.496998063 \mathrm{~kg} \cdot \mathrm{m}^{2} / \mathrm{sec}
$$

If

$$
1 \mathrm{~kg}=1 \mathrm{Kelvin} \text { and for area unit }=1 \mathrm{~m}^{2} \text { and time unit is }=1 \mathrm{sec}
$$

Then

$$
T=-23.496998063 \mathrm{~kg}
$$

In Celsius temperature is

$$
c=k-273.15
$$

$$
\begin{aligned}
& C=-23.496998063-273.15 \\
& T=-296.646998063 \text { Celsius }
\end{aligned}
$$

So almost every particle emits matter and this free matter plays an important role in the expansion of universe. As this free matter is expanded by the right angle triangle theorem in total nothingness.

2) Velocity of Non-intractable Particle with respect to light and black hole Here we will study two velocity factors responsible for expansion of universe:

a) Velocity of light with respect to non intractable particles $\mathbf{v}$ light-non intractable.

b) Velocity of black hole with respect to non intractable particle $\mathbf{v}$ b-ni.

i) The velocity of Non Intractable Particles with respect to light is $299,813,275.22 \mathrm{~m} / \mathrm{sec}$ while the velocity of light is $299,792,458 \mathrm{~m} / \mathrm{sec}$. both light and Non Intractable Particles moves in empty and almost interaction free space due to relativistic quantum effect the light particle continuously move with respect to Non Intractable Particle during this process Matter emits due to gravitational effect between both the particle, which when occupy empty space then matter space and time respectively expand and increase.

Due to this matter and non matter universe increases. The corresponding velocity factor is V light-non intractable $=20,817.22 \mathrm{~m} / \mathrm{sec}$.

ii) Now the velocity of black hole with respect to non intractable particles. This process will also describe that non intractable particles have the potential to 
go beyond black hole. And due to this black holes also move with respect to non intractable particle. This will explain why many black holes are active in the universe.

For this we will use Einstein-bose statistics and consider black hole as a zero of space with almost infinite mass density the equation of bose-einstein statistic is given as $W=\frac{(n i+g i) !}{(n i) ! \cdot(g i) !}$ [5] $\log W=\log (n i+g i) !-\log (n i) !-\log (g i) !$.

Now Using Stirling Approximation $\log n i !=n i \log n i-n i$

$$
\begin{aligned}
& \log W(v)=(n i+g i) \log (n i+g i)-n i \log n i-g i \log g i \\
& \delta(\log W)=(n i+g i) * \frac{1}{(n i+g i)} \cdot \delta n i+\log (n i+g i) \cdot \delta n i \\
&-n i * 1 /(n i)-\log n i \cdot \delta n i \\
& \delta(\log W)(v)=-\log \left(\frac{n i}{n i+g i}\right) \cdot \delta n i
\end{aligned}
$$

For Most Probable Distribution $\delta(\log W \max )=0$, thus the most probable distribution is $\log \left(\frac{n i}{n i+g i}\right) \cdot \delta n i=0$, now taking $\delta(\log W \max )=0 \quad$-(i) 6 according to non-interactive mechanics $N+M=0$.

Equating one and two we get

$$
\delta(\log W \max )=N+M
$$

Here $M$ is $\frac{4 \pi V}{3 h^{3}} \cdot \frac{1}{4} \sqrt{\frac{8 m^{3} \pi}{\beta^{3}}} \cdot e^{\frac{\beta p^{2}}{2 m}}$.

So

$$
\delta(\log \text { Wmax })=N+\left(\frac{4 \pi V}{3 h^{3}} \cdot \frac{1}{4} \sqrt{\frac{8 m^{3} \pi}{\beta^{3}}} \cdot e^{\frac{\beta p^{2}}{2 m}}\right)
$$

Here $\pi=3.14$ taking $V=h^{3}=e^{\frac{\beta p^{2}}{2 m}}=1$.

$$
\delta(\log \text { Wmax })=N+\left(\frac{12.5}{3} \cdot \frac{1}{4} \sqrt{\frac{8 m^{3} \pi}{\beta^{3}}}\right)
$$

Here $n$ is the velocity of non-intractable particle which is $299,813,275.22$ which is $20,817.22 \mathrm{~m} / \mathrm{sec}$ faster than normal detected light photon.

$$
\begin{gathered}
\delta(\log \text { Wmax })=299813275.22+\left(\frac{12.5}{3} \cdot \frac{1}{4} \cdot \frac{2 m}{\beta} \sqrt{\frac{2 m \pi}{\beta}}\right) \\
\delta(\log \text { Wmax })=299813275.22+\left(1.04 \cdot \frac{2 m}{\beta} \sqrt{\frac{2 m \pi}{\beta}}\right)
\end{gathered}
$$

Here $\beta=1 / k T$

$$
\delta(\log W \max )=299813275.22+(1.04 .2 m k T \cdot \sqrt{2 m \pi k T})
$$

if we take temperature as the energy difference between two mass states in Fig- 
ure 17 then

$$
T=2 m(C)^{2}
$$

Then

$$
\begin{gathered}
\delta(\log \text { Wmax })=299813275.22+\left(1.04 \cdot 2 m k \cdot 2 m(C)^{2} \sqrt{2 m \pi k \cdot 2 m(C)^{2}}\right) \\
\delta(\log W \max )=299813275.22+\left(1.04 \cdot 4 \mathrm{~m}^{2} \cdot \mathrm{k} \cdot(C)^{2} \cdot \sqrt{4 m^{2} \pi \mathrm{k} \cdot(C)^{2}}\right) \\
\delta(\log \text { max })=299813275.22+\left(1.04 \cdot 8 \mathrm{~m}^{3} \cdot \mathrm{k} \cdot(C)^{3} \cdot \sqrt{\pi \cdot k}\right) \\
\delta(\log W \max )=299813275.22+\left(1.04 \cdot 8 \mathrm{~m}^{3} \cdot \mathrm{k} \cdot(C)^{3} \cdot \sqrt{\pi \cdot k}\right) \\
\text { for } C=299813275.22 \mathrm{~m} / \mathrm{sec}^{2} \\
K=1.23 \times 10^{-23} \mathrm{~J} / \mathrm{K}, \pi=3.14 \\
\delta(\log W \max )=299813275.22+(1.71395097 \mathrm{E}-8) \\
\delta(\log W \max )=299813275.22+(-4.2759273324) \mathrm{kg} \cdot(\mathrm{m} / \mathrm{sec})^{9} \\
\delta(\log W \max )=299813275.22+(-4.2759273324) \mathrm{kg}^{3} \cdot(\mathrm{m} / \mathrm{sec})^{8} \cdot(\mathrm{m} / \mathrm{sec})
\end{gathered}
$$

For $\mathrm{kg}=1$ and $1 \mathrm{~m} / \mathrm{sec}=10^{-6}$

$$
\begin{gathered}
\delta(\log W \max )=299813275.22+(-4.2759273324) \times\left(10^{-6}\right)^{8}(\mathrm{~m} / \mathrm{sec}) \\
\delta(\log \text { Wmax })=299813275.22+(-4.27592733 \mathrm{E}-48)(\mathrm{m} / \mathrm{sec}) \\
\delta(\log \text { Wmax })=299813275.22+(-57.2907349026)(\mathrm{m} / \mathrm{sec}) \\
\delta(\log \text { Wmax })(v)=299813217.92926 \mathrm{~m} / \mathrm{sec}
\end{gathered}
$$

As $\delta(\log W \max )=0$.

We assume this as a black hole.

So the velocity of black hole is $299,813,217.92926 \mathrm{~m} / \mathrm{sec}$.

So the points are:

a) Velocity of non-intractable particle is $=29,981,3275.22 \mathrm{~m} / \mathrm{sec}$.

b) Velocity of a black hole is $=299,813,217.92926 \mathrm{~m} / \mathrm{sec}$.

c) Velocity of normal detected light is $=299,792,458 \mathrm{~m} / \mathrm{sec}$.

So the results are:

a) Non-intractable particle is $20,817.22 \mathrm{~m} / \mathrm{sec}$ faster than normal detected light.

b) Black hole is $20,759.92926 \mathrm{~m} / \mathrm{sec}$ faster than normal detected light.

c) Non-intractable particle is $57.29074 \mathrm{~m} / \mathrm{sec}$ faster than a black hole.

So whenever a light photon comes under the range of a black it losses itself

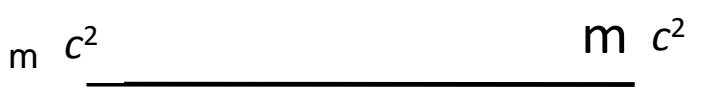

Figure 17. Two mass. 
can't escape from the black hole because normal detected light particle is $20,759.92926 \mathrm{~m} / \mathrm{sec}$ slower than velocity of black hole. This explains due to low velocity light photon can't escape from the black hole.

On the other when a non-intractable particle enters in black it passes through it because non-intractable particle is $57.29074 \mathrm{~m} / \mathrm{sec}$ faster than a black hole. So if a non-intractable particle enter in the black hole it will penetrate and go beyond the attraction and eating range of a black hole.

The corresponding velocity factor is $\mathrm{v} b-\mathrm{ni}=57.29074 \mathrm{~m} / \mathrm{sec}$. Here the velocity factor of black hole to light is also important soV light-b $=20,759.92926$ $\mathrm{m} / \mathrm{sec}$.

Due to these velocity factors non intractable particles, black hole and light ray emit matter and this emitted matter occupies space in the empty universe resultant universe expand continuously.

The resultant velocity factor is

$\mathrm{n}$ (galaxies) $\mathrm{Vga}=\mathrm{n}(\mathrm{V}$ light-Non intractable $+\mathrm{V}$ b-ni $+\mathrm{V}$ light-b).

$\mathrm{n}$ (galaxies) $\mathrm{Vga}=\mathrm{n}(20817.22+57.29074+20759.92926)$.

$\mathrm{n}$ (galaxies) $\mathrm{V} \mathrm{ga}=\mathrm{n}(41634.44) \mathrm{m} / \mathrm{sec}$.

$\mathrm{n}$ (galaxies) is number of Galaxies and $\mathrm{V}$ ga is Velocity of galaxy, Right side $\mathrm{n}$ is Number ofgalaxies Particles.

So for $\mathrm{n}=1$ on both sides velocity of a galaxy is $41,634.44 \mathrm{~m} / \mathrm{sec}$. Thus a galaxy and it's center particle are matter emitter.

3) Particles Entering Rule differences from normal matter to dark matter

The entering rule is the condition for matter or particle to enter in the respective physical field. The entering rule for normal matter universe is given by ramanujan entering rule which is $12 N=-1$ [4] or $12 M=-1$.

For $N=M$ for particle movement is non relativistic.12 $M=-1$ [4].

The entering rule for dark matter is (11.0229152797) $M=-1$ [6].

The difference between both entering is $0.9770847203 M=0$.

Here the value of $\mathrm{M}$ is taken as mentioned above point: (a) (-4.2759273324) $(\mathrm{kg})^{3}-(\mathrm{m} / \mathrm{sec})^{9}$ so the required value is

$$
\begin{aligned}
& 0.9770847203 \times(-4.2759273324)(\mathrm{kg})^{3}-(\mathrm{m} / \mathrm{sec})^{9}=0, \text { for } 1(\mathrm{~m} / \mathrm{sec})=10^{-6} \mathrm{~kg} \\
& 0.9770847203 \times(-4.2759273324)(\mathrm{kg})^{3} \cdot\left((10)^{-6}\right)^{9}=0 \\
& 0.9770847203 \times(-63.2907349026)(\mathrm{kg})^{3}=0 \\
& -61.8404100099(\mathrm{~kg})^{3}=0 .
\end{aligned}
$$

This is always free matter in the universe, more clearly it is the mass value of zero of this universe could be used as mass value of a black hole. Practically this is the mass value of the singularity at the centre of the galaxy. This mass value is free from matter -matter interaction. Thus the particles having this value are free particles and behave as black holes in universe. These particles are two types:

a) Field particles $N+M=0$ [4].

b) Moving particles $\frac{N}{-M}=0$ [4].

"Condition 1 describes the infinite addition property of gravitation."

"Condition 2 describes the infinite dividing property of gravitation." 
The field particles have the velocity of black hole, while the moving particles have the velocity of non-intractable particle. The galaxy rotated by the black hole, the communication and transfer of energy and matter is done by light and expansion of galaxy is done by non intractable particle. This clearly states every galaxy has black hole in its centre even "our milky way galaxy has a black hole in its centre, even our earth has a black hole in its centre" transfer of charge, energy and mass is done by light. And expansion of universe is done by non intractable particle. Every astrophysical object has these three field particle and the mentioned free matter. Even non intractable particles have all three properties of circulation, transfer and Expansion. The practical result of moving particle with free matter can be seen in the link: (https://youtu.be/jfQt-k ueeQ) (Figure 18).

Thus due to universal free matter and three free field particles every astrophysical object expands due to these properties. $M$ entering difference = $0.9770847203 \mathrm{M}$.

\section{4) Converted matter into particle}

This is the well-known fact in the quantum and non interactive mechanics that particles have their existence beyond $0.5 h v$ energy field to $0 h v$ energy field the entering of field quanta from $0 h v$ energy field to $0.5 h v$ energy field happens for the splitting of the elementary particles such as proton and neutron convert into each other with the help of pi mesons (+'ve and -ve). In the reaction where new particles are formed or the parity breaks the production of new types of particles happens such as in the case of productions of k-mesons where strangeness occurs. In these cases new particles occur these particles obtain the matter/energy provided to them as the parity breaks so the gaining of matter by these particles in corresponding field does not obey matter conservation law for stabilizing this condition production of new matter happens by these three properties:

a) Production of new matter by the use of gravitational effect of two relativistic moving non intractable particles with respect to each other.

b) Production on new matter by the use of relativistic quantum system mentioned above.

c) By the use of photo electric effect.

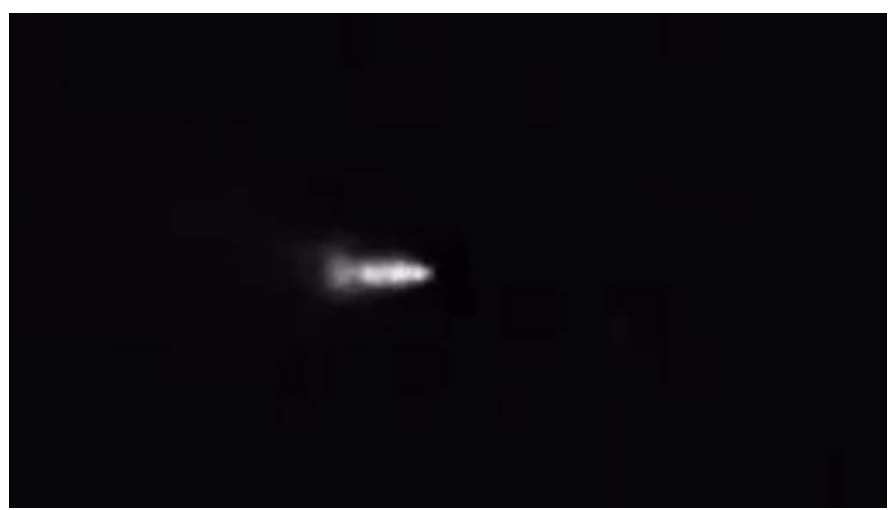

Figure 18. Hubble space telescope. 
So the conversion of matter into particle is also a reason for the expansion of universe. The mathematical form is $\mathrm{m}$ conversion $=\mathrm{n}$ particles.

5) The need of dark energy for expansion of universe

\section{Definition:}

The dark energy is the energy which doesn't lose mass and mechanical energy if working into the field comes under its range, while if it interacts with interactive mass it grows double, Such as: $-1 h v=0.5 h v$. "The Dark matter is the matter which has different entering rule or the matter which has less matter for with respect to normal matter in known world called dark matter. When this matter accelerates with the square of velocity of light is called dark energy". This matter has the value of $0.9770847203 M$.

The basic nature of universe and every object is to expand in all possible manners this work is done through the dark energy as dark energy has the basic nature to expand in all possible manners. The basic restrictions mentioned in the theoretical physics are removed by dark energy in practical universe, as many transformation we are not possible at theoretical level are possible under dark energy as dark energy provides all possible way of reaction, the objects under dark energy can complete reaction tasks with every possibility which we can see in practical life also. This is the reason why many material objects have need of dark energy as they need dark energy for physical actions.

Even in this paper at point No. 10 we showed that $0.5 h v=-1 h_{v}$ so if the normal matter reached to the zero point energy than dark energy becomes as double. Due to this dark matter propagate by the force of gravitation and gravitation waves doubles than normal matter.

After coming under dark energy objects needs dark matter and dark energy to complete task, but due to less mass connection objects looses excess amount of energy into universe and due to mass breakdown of almost $0.9770847203 M$ they can't attach to the universal energy. The statistical value of $m$ dark is

$=\frac{4 \pi V}{3 h^{3}} \cdot \frac{1}{4} \sqrt{\frac{8 m^{3} \pi}{\beta^{3}}} \cdot e^{\frac{\beta p^{2}}{2 m}}$ [4]. Here is a classic example of dark energy expansion.

From Newton's second law of motion

$$
\begin{gathered}
F=d p / d t \\
F=m d v / d t
\end{gathered}
$$

Multiplying with $d s$ on both sides

$$
\begin{gathered}
F \cdot d s=m d v / d t \cdot d s \\
F \cdot d s=m \cdot d v \cdot d s / d t \\
F \cdot d s=m d v \cdot v
\end{gathered}
$$

Integrating both sides

$$
F \cdot s=1 / 2 m \cdot v^{2}
$$

For $s=1$

$$
F=1 / 2 m \cdot v^{2}
$$




$$
\begin{gathered}
F=m a \\
m a=1 / 2 m \cdot v^{2} \\
a=1 / 2 \cdot v^{2} \\
d v / d t=1 / 2 \cdot v^{2} \\
v^{-2} d v=1 / 2 d t
\end{gathered}
$$

Integrating on both sides we have

$$
\begin{gathered}
v^{-1} /-1=1 / 2 d t \\
-1=1 / 2\left(v^{-1}\right) \cdot d t \\
-1=v / 2 d t \\
-1=(d x / d t \cdot 2) \cdot d t \\
-1=d x / 2
\end{gathered}
$$

Integrating right hand side with respect to $d x$

$$
-1=x / 2)
$$

For $x=1$

$$
-1=0.5
$$

Multiplying both sides by $h v$

$$
-h v=0.5 h v
$$

This shows zero point energy link with dark energy and negative energy. This shows whenever a linear harmonic oscillator oscillates they gain dark energy or negative energy. This is the reason that almost $70 \%$ energy of universe is dark and lots of matter is dark matter.

Some Basic conversation of Particles and matters in dark energy are:

a) Condition $N=M$, this condition is used only when the motion of objects are non relativistic.

b) $N=M^{2}$, this condition is used when the two mass bodies are in the relativistic motion.

c) $N= \pm 1$, this condition describes the emission of particle, $N=+1$ for emission of non intractable particle beyond the field of the periodically simulating space time, and $N=-1$ describe the emission of non intractable particle in periodically simulating space-time.

d) $N=0$ describes that non intractable particle is not present in the matter field.

e) $M= \pm 1$ describe the rate of matter-particle interaction and the direction of reaction $m=+1$ for the condition where total energy is less than the sum of kinetic and potential energy and $m=-1$ for the condition where total energy is higher than the sum of kinetic and potential energy. This condition is explained in the paper on the development of the photo electric effect.

f) $M=0$ this condition describes the emptiness of universe space-time which 
is not filled by matter.

g) Velocity of light $C=i$.

h) his condition is present when the non-intractable particle is not present in the matter field or the non intractable particle at rest, as the mass of non intractable particle and light are same but the velocities are different so for the condition when the non intractable particle either at rest or not present in the matter field the velocity of light becomes the highest in the matter field and the difference of velocity of light with other objects becomes as high that it becomes imaginary for all the objects moving relative to it.

This dark energy expands the universe in all possible manners. So the required factor is $M$ dark. So the resultant factor for the expansion of universe is Expansions factor $\mathrm{U}$ e. $\mathrm{U}$ e $=\mathrm{M}$ free $+\mathrm{n}$ (galaxies) $\mathrm{V}$ ga $+\mathrm{M}$ entering difference $+\mathrm{M}$ conversion $+\mathrm{M}$ dark $=2 \mathrm{~T} /(\mathrm{C})^{2}+\mathrm{n}(\mathrm{V}$ light-Non intractable $+\mathrm{V}$ b-ni $+\mathrm{V}$ light-b) $+0.9770847203 \mathrm{M}+\mathrm{N}$ particle $+\mathrm{M}$ dark.

These are 7 Basic factors for expansion of universe.

\section{Black Body Radiation in Expanding Universe}

The case of a perfect black body is very important, practically by a black body we are talking about which can found his existence in the expanding and cmbr universe. The case of perfect black body is following all the basic rules of modern physics but the terms are expanded more.

Starting with the basics

$$
V=C / \lambda
$$

For $C=g i e^{\alpha+\beta E i}-e^{-\alpha-\beta E i}$ according to non interactive mechanics

$$
V=\frac{g i e^{\alpha+\beta E i}-e^{-\alpha-\beta E i}}{\lambda}
$$

If $\lambda$ is defined for a charge wave particle, which has space creation property in that case for $\lambda$ is using space creation rule of cosmological concept of $N \mathrm{I} \mathrm{Me}$ chanics [3]

$$
\left(\frac{N}{-\frac{4 \pi V}{3 h^{3}} \cdot \frac{1}{4} \sqrt{\frac{8 m^{3} \pi}{\beta^{3}}} \cdot e^{\frac{\beta p^{2}}{2 m}}}\right)^{2 \cdot n}=2 \cdot n \frac{N}{-\frac{4 \pi V}{3 h^{3}} \cdot \frac{1}{4} \sqrt{\frac{8 m^{3} \pi}{\beta^{3}}} \cdot e^{\frac{\beta p^{2}}{2 m}}}
$$

Using R H S of above equation in place of $\lambda$

$$
\begin{gathered}
V=\frac{g i e^{\alpha+\beta E i}-e^{-\alpha-\beta E i}}{\lambda} \\
2 \cdot n \frac{g i e^{\alpha+\beta E i}-e^{-\alpha-\beta E i}}{N} \\
-\frac{4 \pi V}{3 h^{3}} \cdot \frac{1}{4} \sqrt{\frac{8 m^{3} \pi}{\beta^{3}}} \cdot e^{\frac{\beta p^{2}}{2 m}}
\end{gathered}
$$




$$
\begin{gathered}
V=\frac{g i e^{\alpha+\beta E i}-e^{-\alpha-\beta E i}}{2 \cdot n \frac{N}{-\frac{4 \pi V}{3 h^{3}} \cdot \frac{1}{4} \sqrt{\frac{8 m^{3} \pi}{\beta^{3}}} \cdot e^{\beta E i}}} \\
V=\frac{-\frac{\pi V}{3 h^{3}} \sqrt{\frac{8 m^{3} \pi}{\beta^{3}}} \cdot e^{\beta E i}\left(g i e^{\alpha+\beta E i}-e^{-\alpha-\beta E i}\right)}{2 \cdot n N} \\
V=\frac{-\frac{\pi V}{3 h^{3}} \sqrt{\frac{8 m^{3} \pi}{\beta^{3}}} \cdot e^{\beta E i}\left(g i e^{\alpha+2 \beta E i}-e^{-\alpha}\right)}{2 \cdot n N}
\end{gathered}
$$

For $e^{-\alpha}=$ constant

$$
\begin{gathered}
V=\frac{-\frac{\pi V}{3 h^{3}} \sqrt{\frac{8 m^{3} \pi}{\beta^{3}}} \cdot\left(g i e^{2 \beta E i}\right)}{2 \cdot n N} \\
V=\frac{-\frac{\pi V}{3 h^{3}} \sqrt{\frac{8 m^{3} \pi}{\beta^{3}}} \cdot\left(g i e^{2 \beta E i}\right)}{N \cdot 2 n} \\
V=\frac{-\pi V \sqrt{8 \pi m^{3}}(k T)^{3} \cdot\left(g i e^{2 \beta E i}\right)}{N \cdot 3 h^{3} \cdot 2 \cdot n} \\
V=\frac{-\pi V \sqrt{8 \pi m^{3}}(k \cdot T)^{3} \cdot\left(g i e^{2 \beta E i}\right)}{N \cdot 3 h^{3} \cdot 2 \cdot n} \\
V=\frac{-\pi V \sqrt{2 \pi m^{3}}(k \cdot T)^{3} \cdot\left(g i e^{2 \beta E i}\right)}{N \cdot 3 h^{3} \cdot n}
\end{gathered}
$$

for $n=1$

$$
V=\frac{-\pi V \sqrt{2 \pi m^{3}}(k \cdot T)^{3} \cdot\left(g i e^{2 \beta E i}\right)}{N \cdot 3 h^{3}}
$$

Now using Max plank's frequency rule

$$
E v \cdot d v=\frac{8 \cdot \pi \cdot h(v)^{3} \cdot d v}{c^{3} \cdot e^{h v / k T}-1}
$$

Substituting the value of $v$ from above equation

$$
\begin{gathered}
V=\frac{-\pi V \sqrt{2 \pi m^{3}}(k \cdot T)^{3} \cdot\left(g i e^{2 \beta E i}\right)}{N \cdot 3 h^{3}} \\
E v \cdot d v=\frac{8 \cdot \pi \cdot h\left(\frac{-\pi V \sqrt{2 \pi m^{3}}(k \cdot T)^{3} \cdot\left(g i e^{2 \beta E i}\right)}{N \cdot 3 h^{3}}\right)^{3} \cdot d v}{c^{3} \cdot e^{h \frac{-\pi V \sqrt{2 \pi m^{3}}(k \cdot T)^{3} \cdot\left(g i e^{2 \beta E i}\right)}{N \cdot 3 h^{3}} / k T}-1}
\end{gathered}
$$




$$
\begin{gathered}
E v \cdot d v=\frac{8 \cdot \pi \cdot h\left(\frac{-\pi V \sqrt{2 \pi m^{3}}(k \cdot T)^{3} \cdot\left(g i e^{2 \beta E i}\right)}{N \cdot 3 h^{3}}\right)^{3} \cdot d v}{c^{3} \cdot\left(1+\frac{-\pi V \sqrt{2 \pi m^{3}}(k \cdot T)^{3} \cdot\left(g i e^{2 \beta E i}\right) \cdot h}{N \cdot 3 h^{3} \cdot k T}\right)-1} \\
=\frac{8 \cdot \pi \cdot k T\left(\frac{-\pi V \sqrt{2 \pi m^{3}}(k \cdot T)^{3} \cdot\left(g i e^{2 \beta E i}\right)}{N \cdot 3 h^{3}}\right)^{2} \cdot d v}{c^{3}} \\
=\frac{8 \cdot \pi \cdot k T\left(-\pi V \sqrt{2 \pi m^{3}}(k \cdot T)^{3} \cdot\left(g i e^{2 \beta E i}\right)\right)^{2} \cdot d v}{9 C^{3} h^{6} \cdot N^{2}} \\
=\frac{8 \cdot \pi \cdot k T \cdot\left(\pi^{2} V^{2} 2 \pi m^{3} \cdot(k \cdot T)^{6} \cdot\left(g i e^{4 \beta E i}\right)\right)}{9 C^{3} h^{6} N^{2}} \cdot d v \\
E v \cdot d v=\frac{16\left(\pi^{4} V^{2} m^{3} \cdot(k \cdot T)^{7} \cdot\left(g i e^{4 \beta E i}\right)\right)}{9 C^{3} h^{6} N^{2}} \cdot d v \\
=\frac{16\left(\pi^{4} V^{2} m^{3} \cdot(k \cdot T)^{7} \cdot(g i)^{2}\left(1+4 \beta E i+16(\beta E i)^{2}+64(\beta E i)^{3}\right)\right)}{9 C^{3} h^{6} N^{2}} \cdot d v
\end{gathered}
$$

This is the Black body radiation rule in terms of frequency.

For $T=2 m c^{2}$

$E v \cdot d v=\frac{16 c^{14}\left(\pi^{4} V^{2} m^{3} \cdot(k \cdot 2 m)^{7} \cdot(g i)^{2}\left(1+4 \beta E i+16(\beta E i)^{2}+64(\beta E i)^{3}\right)\right)}{9 C^{3} h^{6} N^{2}} \cdot d v$

$E v \cdot d v=\frac{-13974.994639866\left(V^{2} \cdot(m)^{10}(g i)^{2}\left(1+4 \beta E i+16(\beta E i)^{2}+64(\beta E i)^{3}\right)\right)}{9 h^{6} N^{2}} \cdot d v$

$E v \cdot d v=\frac{-1522.7771822096\left(V^{2} \cdot(m)^{10}(g i)^{2}\left(1+4 \beta E i+16(\beta E i)^{2}+64(\beta E i)^{3}\right)\right)}{h^{6} N^{2}} \cdot d v$

According to developed photo electric effect $N$ has the value of

$$
N=19 \times 10^{-10} \mathrm{Gev} \cdot \mathrm{sec} / c^{2}
$$

In $\mathrm{kg}$ this value is $N=-28.61248 \mathrm{~kg}$ putting in above eq.

$$
E v \cdot d v=\frac{1.8600531571\left(V^{2} \cdot(m)^{10}(g i)^{2}\left(1+4 \beta E i+16(\beta E i)^{2}+64(\beta E i)^{3}\right)\right)}{h^{6}} \cdot d v
$$

\section{Equation of Black body in Wavelength term}

The above equation of black body is

$$
E v \cdot d v=\frac{16\left(\pi^{4} V^{2} m^{3} \cdot(k \cdot T)^{7} \cdot(g i)^{2}\left(1+4 \beta E i+16(\beta E i)^{2}+64(\beta E i)^{3}\right)\right)}{9 C^{3} h^{6} \cdot N^{2}} \cdot d v
$$




$$
\begin{aligned}
& \text { For } V=C / \lambda \text { and } d v=-\left(\frac{C}{\lambda}\right)^{2} \cdot d \lambda \\
& E \lambda \cdot d \lambda=\frac{16\left(\pi^{4} V^{2} m^{3} \cdot(k \cdot T)^{7} \cdot(g i)^{2}\left(1+4 \beta E i+16(\beta E i)^{2}+64(\beta E i)^{3}\right)\right)}{9 C^{3} h^{6} \cdot N^{2}} \\
& \cdot\left|-C / \lambda^{2}\right| \cdot d \lambda u^{\prime} \\
& \text { For } C=g i e^{\alpha+\beta E i}-e^{-\alpha-\beta E i} \text { and } \lambda=2 \cdot n \frac{N}{-\frac{4 \pi V}{3 h^{3}} \cdot \frac{1}{4} \sqrt{\frac{8 m^{3} \pi}{\beta^{3}}} \cdot e^{\frac{\beta p^{2}}{2 m}}} \\
& E \lambda \cdot d \lambda=\frac{16\left(\pi^{4} V^{2} m^{3} \cdot(k \cdot T)^{7} \cdot(g i)^{2}\left(1+4 \beta E i+16(\beta E i)^{2}+64(\beta E i)^{3}\right)\right)}{9 C^{3} h^{6} \cdot N^{2}} \\
& \left(2 \cdot n \cdot \frac{g i e^{\alpha+\beta E i}-e^{-\alpha-\beta E i}}{\left(\frac{-4 \pi V}{3 h^{3}} \cdot \frac{1}{4} \sqrt{\frac{8 m^{3} \pi}{\beta^{3}}} \cdot e^{\frac{\beta p^{2}}{2 m}}\right)^{2 \cdot n}}\right)
\end{aligned}
$$

For $n=1 / 2$

$$
\begin{gathered}
E \lambda \cdot d \lambda=\frac{16\left(\pi^{4} V^{2} m^{3} \cdot(k \cdot T)^{7} \cdot(g i)^{2}\left(1+4 \beta E i+16(\beta E i)^{2}+64(\beta E i)^{3}\right)\right)}{9 C^{3} h^{6} \cdot N^{2}} \\
E \lambda \cdot d \lambda=\frac{8\left(\pi^{3} V^{2} m^{2} \cdot(k \cdot T)^{6} \cdot(g i)^{2}\left(1+4 \beta E i+16(\beta E i)^{2}+64(\beta E i)^{3}\right)\right)\left(g i-e^{-2 \beta E i}\right)}{3 \cdot \pi V \sqrt{8 \pi m^{3}}(k \cdot T)^{3}} \cdot d \lambda \\
3 C^{3} h^{3} \cdot N^{3} \sqrt{2 \pi m k t}
\end{gathered}
$$

Here $h$ is degrees of freedom.

This is the law of radiation for black body in terms of wavelength. The values of plank constant can be calculated by the process mentioned above for different Mass Bodies using the above relations. Placing the value of $v$ cab be evaluated by the above relation and the value of $\mathrm{E}$ can be obtained for two mass and charge body by the use of relation $E=M C^{2}$.

Then by use of $E=h v$ and $E=M C^{2}$.

Equating both we have

$$
h v=M C^{2}
$$

Then $h=M C^{2} / v$

$$
V=\frac{-\pi V \sqrt{2 \pi m^{3}}(k \cdot T)^{3} \cdot\left(g i e^{2 \beta E i}\right)}{N \cdot 3 h^{3}}
$$




$$
\begin{aligned}
M n-I & =13.6160 \mathrm{Gev} \cdot \mathrm{sec} / C^{2} \\
& =13.4160 \times 1.79 \times 10^{-27} \\
& =-20.782107712 \mathrm{~kg}
\end{aligned}
$$

For $E=M C^{2}=-20.782107712 \times(299813275.22)^{2}$ and

$$
v=\frac{-\pi V \sqrt{2 \pi m^{3}}(k \cdot T)^{3} \cdot\left(g i e^{2 \beta E i}\right)}{N \cdot 3 h^{3}} \text {. }
$$

For $V=1, N=1, h=1$ and $g i e^{2 \beta E i}=1$

$$
V=-17.9924131466 \mathrm{sec}
$$

Negative frequency shows same result as derivation and numerical values.

For $E=h v$

$$
\begin{gathered}
h=E / v=M n i \times C^{2} / v \\
h=13.9410746691 /(-17.9924131466) \\
h=-0.7748307331 \mathrm{~J} \cdot \mathrm{sec}
\end{gathered}
$$

Using non interactive Mechanics

Dark energy for particles moving with the velocity of non-intractable particle and following Ramanujan Entering.

$$
V=-17.9924131466 \mathrm{sec}
$$

For $-1=12 N$

$$
V=12 N \times(-17.9924131466)
$$

For $N=-M$

$$
V=12 \times(-M) \times(-17.9924131466)
$$

(For $M=-57.2907349026)$

$$
\begin{gathered}
V=12 \times(-57.2907349026) \times(-17.9924131466) \\
V=12369.582862079 \mathrm{sec}
\end{gathered}
$$

From $h=\frac{M C^{2}}{v}$

$$
\begin{gathered}
M C^{2}=-57.2907349026 \times 299813275.22 \times 299813275.22 \\
=6.8106241126 \mathrm{~kg} \cdot \mathrm{m}^{2} / \mathrm{sec}^{2} \\
h=6.8106241126 / 12369.582862079 \\
h=0.0053644581 \mathrm{~J} \cdot \text { Second }
\end{gathered}
$$

These are the values for non intractable particles, similarly value of $h$ can be evaluated for different Mass Bodies for quantum and non interactive cases.

Dark energy plank constant for particles moving with the velocity of Non-intractable particle and following Ramanujan Entering.

$$
\begin{gathered}
V=-17.9924131466 \mathrm{sec} \\
(11.0229152797) M=-1[6]
\end{gathered}
$$

The value of $v$ is 


$$
\begin{gathered}
V=-17.9924131466 \\
=17.9924131466 \times(11.0229152797) M \\
=17.9924131466 \times(11.0229152797) \times(-57.2907349026) \\
(\text { For } M=-57.2907349026) \\
=-11362.405327827 \mathrm{sec}
\end{gathered}
$$

Now for

$$
\begin{gathered}
h=\frac{M C^{2}}{v} \\
M C^{2}=6.8106241126 \mathrm{~kg} \cdot \mathrm{m}^{2} / \mathrm{sec}^{2} \\
h=-77.385 .071702833 \mathrm{~kg} \cdot \mathrm{m} / \mathrm{sec}^{2}
\end{gathered}
$$

without non interactive mechanics

$$
\begin{gathered}
V=-17.9924131466 \\
h=\frac{M C^{2}}{v}
\end{gathered}
$$$$
M C^{2}=-57.2907349026 \times 299792458 \times 299792458=-0.3786139107
$$

First $M=-1$ then $M=+1$

$$
h=0.3786139107 \mathrm{~kg} \cdot \mathrm{m} / \mathrm{sec}^{2}
$$

So For Ramanujan Entering the value of plank constant are

$$
h=-0.7748307331 \mathrm{~J} \cdot \mathrm{sec}
$$

For $M=-1$ then $M=+1$

$$
\begin{gathered}
h=0.7748307331 \mathrm{~J} \cdot \mathrm{sec} \\
h=0.0053644581 \mathrm{~J} \cdot \text { Second }
\end{gathered}
$$

Dark Energy entering rules and values of $M$. Plank constant according to non interactive mechanics

$$
h=-77385.071702833
$$

For $M=-1$ then $M=+1$

$$
\begin{gathered}
h=77385.071702833 \mathrm{~J} \cdot \mathrm{sec} \\
h=0.3786139107 \mathrm{~J} \cdot \mathrm{sec}
\end{gathered}
$$

Values II and IV are the quantum values of relativistic quantum figure. While eq. I and III are the relativistic part of relativistic quantum figure.

\section{Nature of Universe: The Mathematical Tools Used by Universe}

The equation of 0 can be defined by these 2 condition:

1) First field stable zero $N+M=0$.

2) Second movable zero $N /(-M)=0$.

Equating both sides $N+M=N /(-M)$. 


$$
\begin{gathered}
M^{2}+N M+N=0 \\
M(N+M)+N=0 \\
\text { For } \quad N+M=0 \\
N+0=0 \\
N+N+M=0
\end{gathered}
$$

1) $2 N+M=0$

$$
\begin{gathered}
N+0=0 \\
N+N /(-M)=0 \\
N=N \cdot M
\end{gathered}
$$

2) $M=1$

$$
N+N /(-M)=0
$$

Interchanging $N$ and $-M$ on numerator and denominator

$$
\begin{gathered}
N+N /(-M)=0 \\
N=-M \\
-M+(-M / N)=0 \\
-M / N=M \\
-1 / N=1 \\
N=-1
\end{gathered}
$$

3) $N=-1$

\section{So four results are:}
1) $2 N+M=0$
2) $M=1$
3) $N=-1$
4) $N^{2}=M$

\section{Condition derived by these equations:}

1) $2 N+M=0$

For $M=1$

$2 N+1=0$

$N=-1 / 2$

Non in tractable particle has the spin of $-1 / 2$

2) $2 N+M=0$

For $M=N^{2}$

$2 N+N^{2}=0$

$N=-2$

For attending non interactive particle axial range should be $N=-2$

3) $2 N+M=0$

For $N=-1$

$-2+M=0$

$M=2$ 
For attending non intractable matter the range should be $M=2$

For $N+M=0$

So the condition of (2) and (3) Properly fit in the non interactive mechanics.

For totally class zero particles:

Resultant values

1) For $N+M=0$

$N=-1 / 2$ and $M=2$

$-1 / 2+2=0$

$3 / 2=0$

Class zero particles have the spin of $3 / 2$. So if a particle have the spin of $3 / 2$ it must be a class zero particles. $3=0$.

Now the number of particle giving displacement and movement are 3.

For $N+M=0$

2) $\quad N=-1 / 2$ and $M=1$

$-1 / 2+1=0$

$1 / 2=0$

Class zero particle has the spin of $1 / 2$

(For $N=-M,-1 / 2=-1,1 / 2=1$ )

After displacement from zero to 1 axially the spin still $1 / 2$.

3) $\quad N+M=0$

For $N=-2$ and $M=-2$

$2-2=0$

$0=0$

Non interactive mechanics, non intractable particle and non intractable matter follow universe and mathematical rules. Means to say if we cut a class zero particle similar class zero particle emerge with same qualities.

4) $\quad N+M=0$

For $N=-2$ and $M=1$

$-2+1=0$

$-1=0$

Class zero particle and its field have connection in energy form.

Properties of Non Intractable Particles

Non Intractable Particles have the left hand side rotation $(N=-1 / 2)$, Non Intractable particles have two types $(N=-2)$. Matter field also divides into two types $(M=2)$. Which is same as non interactive Mechanics.

\section{Class Zero Field Properties}

In pure class zero field:

1) Three class zero particles are required for production of pure class zero field.

2) Class zero particles have the spin of integral half spin, it can be either right or left hand side rotation. But as non interactive mechanics says positive geometry and right hand side rotation are prime and first spin so the class zero particle 
has first right hand side rotation or positive rotation. So class zero particles have $+1 / 2$ rotation.

3) Class zero fields are identical no distinguishability in two-class zero field only when matter is not emitted.

4) Class zero field is backhanded by negative energy field or class zero field holds the situation to get energy from dark energy field. These are some properties of non Intractable Particles and class zero particles.

\section{Scope for the Expansion of Universe}

The Relativistic Quantum mechanical condition stated as $i \hbar \partial \psi / d t= \pm \sqrt{(P c)^{2}}+\left(m c^{2}\right)^{2}$. Now here we try to interpret the wave structure and properties presented by this equation by considering each side and its relative terms, Let us consider first L.H.S.

\section{L.H.S ( $i \hbar \partial \psi / d t)$ :}

1) The Q. Mech. Wave $\psi$, as many theories and experiments predicted vary qualities of waves with different structures such as circular, spherical wave, but the most common structure for a wave considered as spherical, the reason behind choosing the spherical shape as many of atomic model theories successfully predicted the spherical shape of atomic orbital's which was confirmed by experimental proofs, except their an exception for hydrogen atom which presents a circular wave. So for a normal condition the representative wave taken as spherical (Figure 19).

2) Now considering the differentiable part is $(\partial / d t)$, now this represents two parts.

a) The wave is differentiating w.r.t or moving under time $t$.

b) If we write it as ( $i \partial / d t)$, then it can be written as $\left(i^{2} c \partial \psi / \partial(i c t)\right)$.

So by the first view the first diffr. W.r.t to time $(t)$, looks as the fraction in Figure 20 of above presented wave.

As if look the wave then and study its geometry than for space and time it represents the Riemannian geometry with the equation Euclidean geometry $\left(d s^{2}=d X u d X u g u v\right)$.

Now as predicted by Einstein the curve geometry turned into straight geometry in special condition or the case of Riemannian geometry turned into Euclidean geometry (Euclidean geometry $\left(\mathrm{ds} s^{2}=d x 1^{2}+d x 2^{2}+d x 3^{2}+d x 4^{2}\right)$ here ( $\left.X 4=i c t\right)$

In normal conditions the curve wave of Riemannian geometry looks as straight line wave of Euclidean geometry. Riemannian geometry $d s^{2}=d X u d X u$ guv Euclidean geometry $\left(\mathrm{ds} s^{2}=d x 1^{2}+d x 2^{2}+d x 3^{2}+d x 4^{2}\right)$. Here the $\hbar$ represents the minimum required energy per unit time for wave which is $\hbar=4.2203 * 10^{-34}$. So the wave equation has the form of $(-c \hbar \partial \psi / \partial i c t)$. Actual geometry seems as Figure 21.

The remaining Term representing the movement of given wave with respect to absolute velocity $C$ speed of light. Now looking at the right hand side of the Equation (1) 


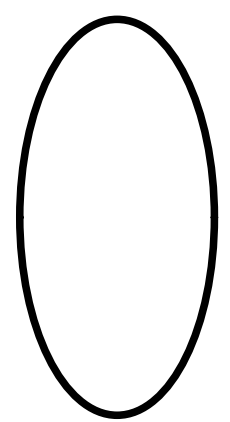

Figure 19. $\psi$ normal condition the representative wave.

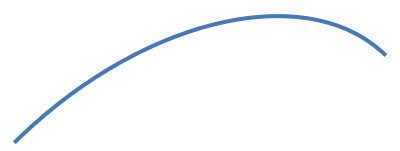

Figure 20. $\partial \psi / d t$.

\begin{tabular}{l|l}
$\boldsymbol{\psi}$ & $\begin{array}{c}\left(\hbar=4.2203 * 10^{-34} \mathrm{~J}-\mathrm{s}\right) \\
-\mathrm{c} \hbar / \mathrm{dt}\end{array}$
\end{tabular}

Figure 21. Actual geometry.

\section{R.H.S:}

$\pm \sqrt{(P c)^{2}}+\left(m c^{2}\right)^{2} \psi$ the part deal with the energy distribution in above shown wave.

Now if we assume that this shows the wave similar as L.H.S than let's just see the distribution of energy in the above wave $\pm \sqrt{m^{2}} \cdot c^{2}+(P c)^{2} \psi$, Now using binomial theorem $\pm \sqrt{(P c)^{2}}+\left(m c^{2}\right)^{2} \psi= \pm\left(1+1 / 2 !\left(\left(m c^{2}\right)^{2}+(P c)^{2}\right)\right) \quad$ (Here neglecting higher power term) so the Equation (1) looks as $i \hbar \partial \psi / d t= \pm\left(1+1 /\left(\left(m c^{2}\right)^{2}+(P c)^{2}\right)\right) \psi$ the form of sign distribution is ignored till final Figure 22 arrive. And the energy distribution given above. Now the second part of above line as: $\pm \sqrt{m^{2} c^{4}}+(P C)^{2} \cdot \psi$, now the part deal with the energy distribution in above shown wave.

Now if we assume that this shows the wave similar as L.H.S than let's just see the distribution of energy in the above wave $\pm \sqrt{m^{2} c^{4}}+(P c)^{2} \cdot \psi$, now using binomial theorem $\pm \sqrt{m^{2} c^{4}}+(P c)^{2} \cdot \psi= \pm\left(1+\frac{1}{2} !\left(\left(m c^{2}\right)^{2}+(P c)^{2}\right)\right)$ (Here neglecting higher power term) so the Equation (1) looks as: $\frac{i \hbar \partial \psi}{d t}= \pm\left(1+\frac{1}{2} !\left(\left(m c^{2}\right)^{2}+(P c)^{2}\right)\right) \psi$.

Now if substituting $P=-i \hbar \partial \psi / d x$ and distributing "signs" on Figure 23 then the figure shown above justify the sign balancing of upper quantum state and lower classical state and as we can see that shown figure justify that both 


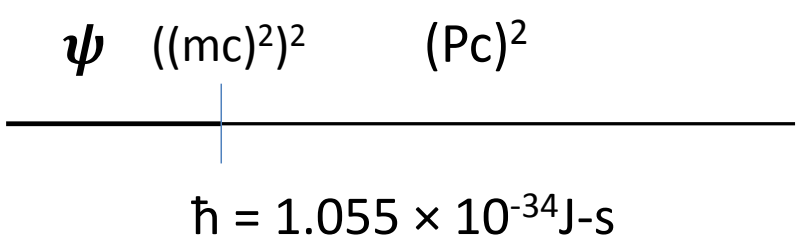

Figure 22. The form of sign distribution.

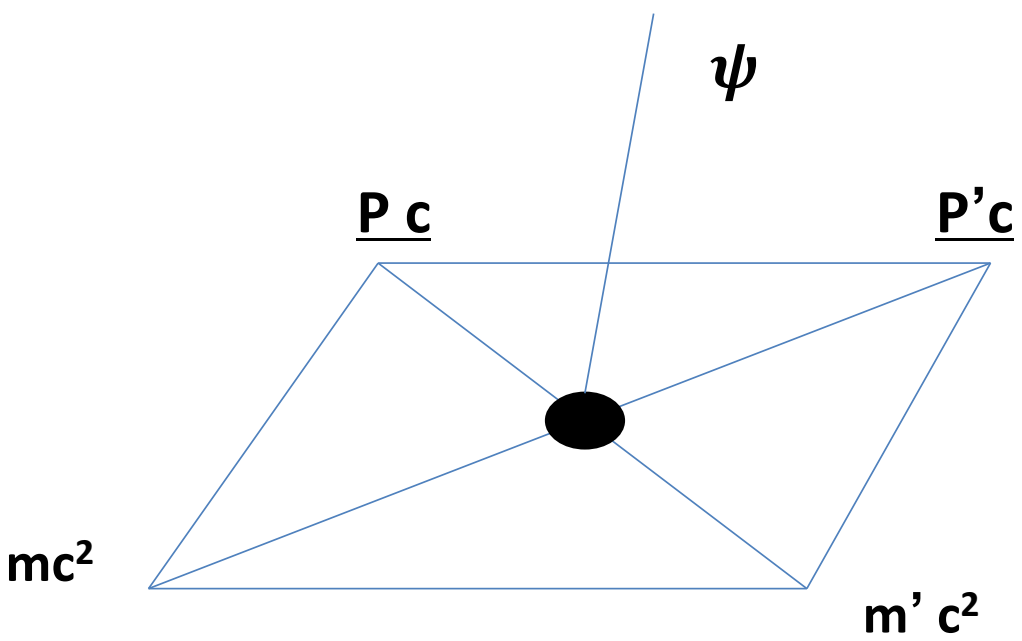

Figure 23. Sign balancing of upper quantum state and lower classical state.

classical energy state and quantum energy state is bound in a same figure and the mass varies relatively (as $m, m^{\prime}$ ) so the case of classical mechanics and quantum mechanics are in same figure and their mass vary relatively with a perfect black body at center which justify the condition.

Plank (Quantum) $<<$ Newton (classical) $>>$ Einstein (Relativity)

Now for charge distribution above figure is

Quality of these kinds of waves:

1) Relativistic Energy $E n=M \cdot C^{2} \cdot n$.

2) Quantum Energy $E=n \cdot h v$.

3) Waves motion type $=$ linear harmonic oscillator.

4) Velocity = velocity of light and Non-intractable particles.

5) Waves momentum energy relation $E^{2}=P^{2} \cdot C^{2}+M^{2} \cdot C^{4}$.

Figure 24 shown down there is for Euclidean high energy physics and so the lines are straight. Then we show the Riemannian geometry case of low energy physics they are shown as Figure 25, Figure 26.

This is the case of Riemannian geometry and low energy physics. According to current research these points can't be explained without the condition as Figure 27:

Here $\psi_{R}$ has the velocity $=299,813,275.22 \mathrm{~m} / \mathrm{s}$.

Here $\psi_{x}$ has the velocity $=299,792,458 \mathrm{~m} / \mathrm{s}$.

Here $\psi_{w}$ has the velocity $=299,813,217.92926 \mathrm{~m} / \mathrm{sec}$.

Corresponding waves figures are as follows: Figure 28, Figure 29. 


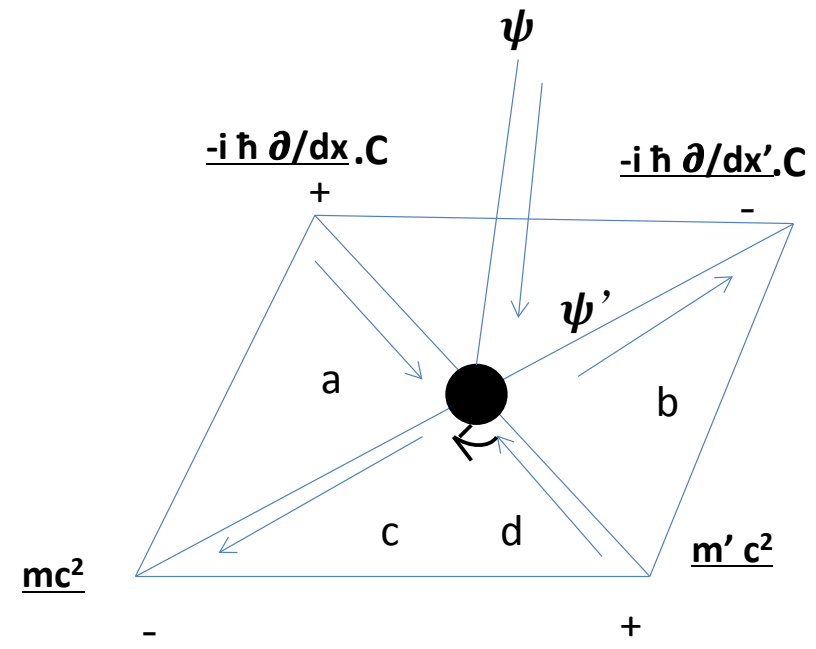

Figure 24. Charge distribution.

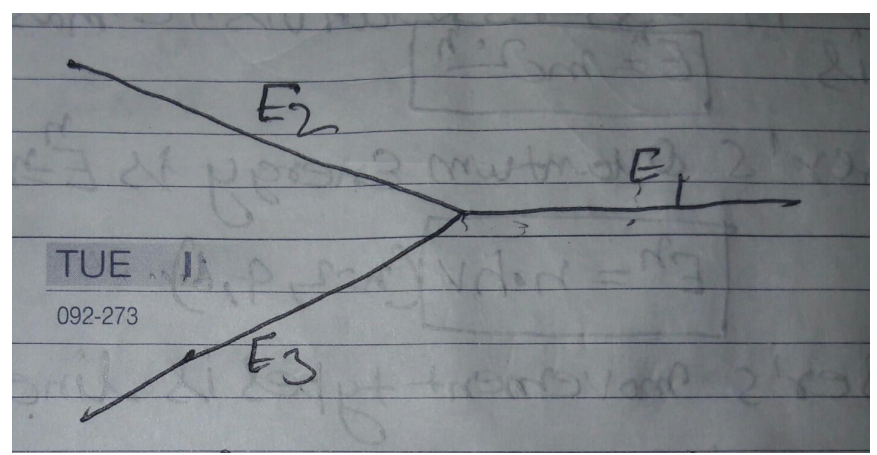

Figure 25. This is the case Euclidean geometry at high energy physics.

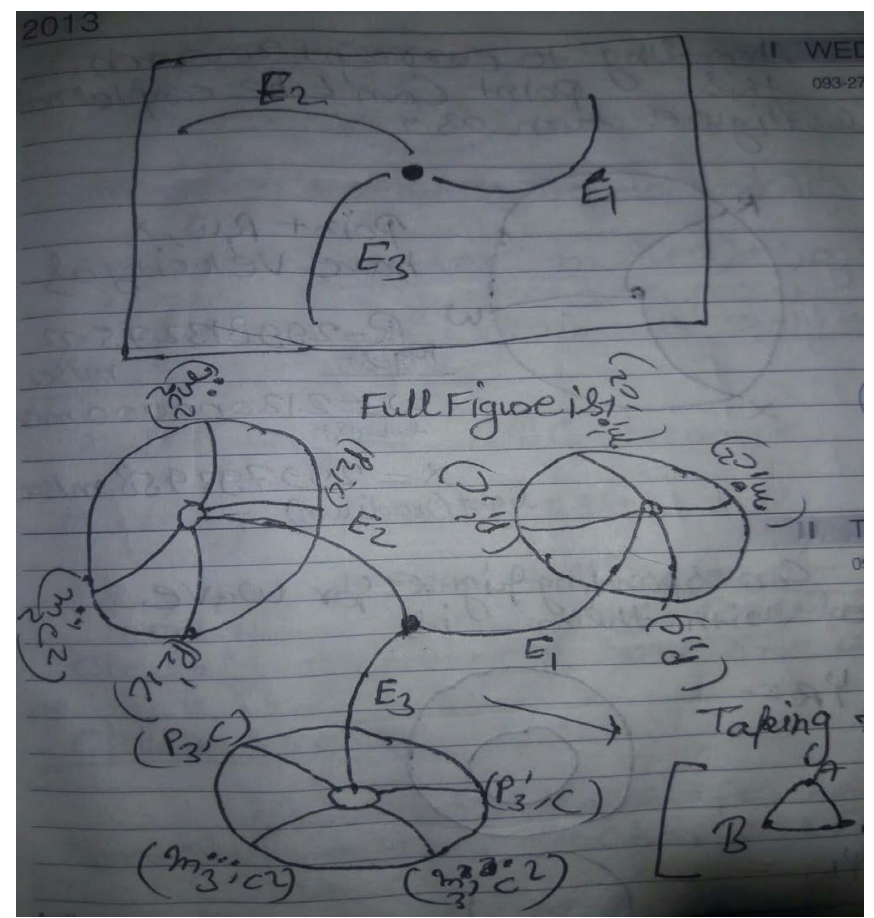

Figure 26. Riemannian geometry at low energy level. 


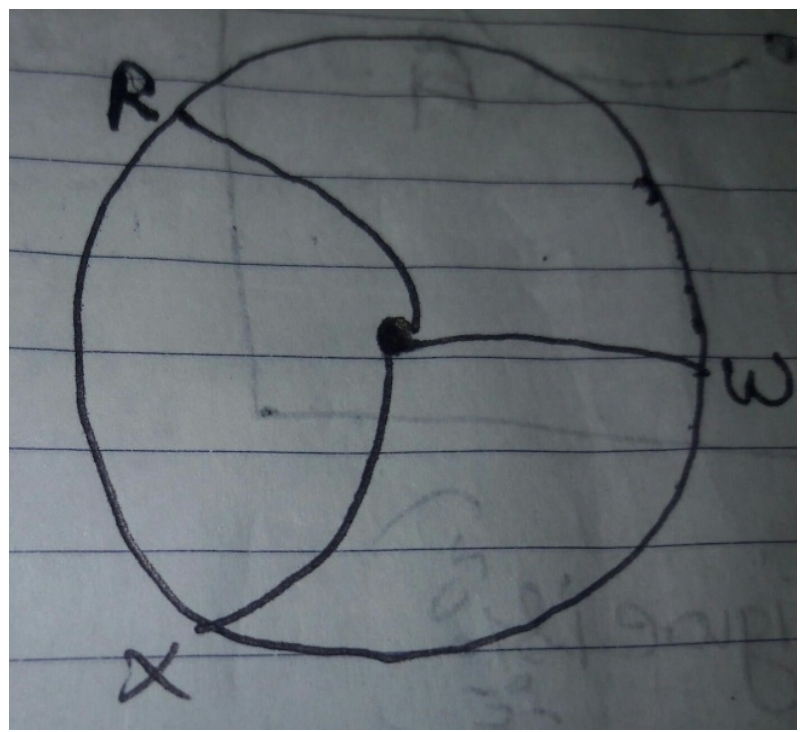

Figure 27. Condition.

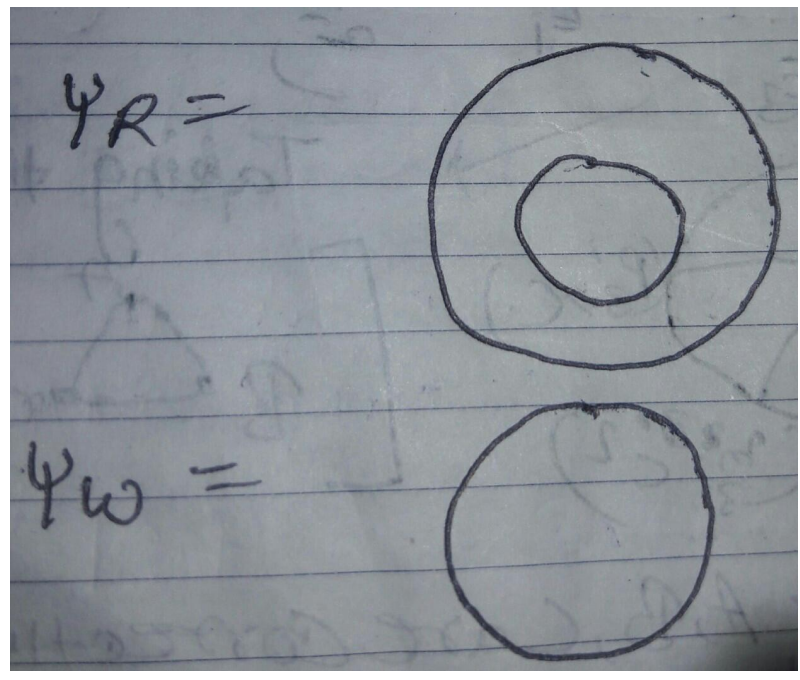

Figure 28. Plasma figure of the $\psi_{R}$ and $\psi_{W}$ in plasma condition at different waves.

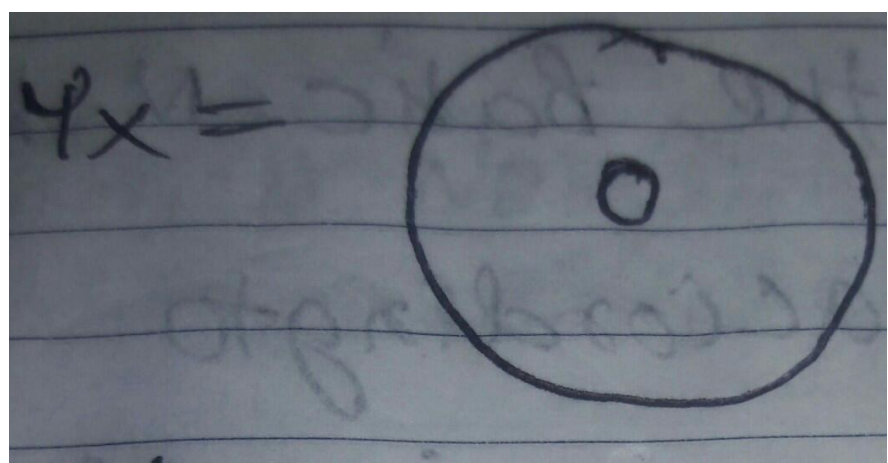

Figure 29. Plasma condition of Figure 15 at the velocity of light.

According to Quantum Mechanics and azimuthal quantum number $M=+2$, $+1,0,-1,-2$. But according to non interactive mechanics and practically may be 
only three figures are possible. But even this condition of nothing will appear unless these forms and basic structure of reaction as appear in the spark chamber. To find the final structure of matter we have to understand the nature of zero.

The equation of 0 can be defined by these 2 conditions:

1) First field stable zero $N+M=0$ where Non-intractable particles makes union with Matter.

2) Second movable zero $N I(-M)=0$ where continuous emission of Non Intractable Particles.

In form of quanta with respect to Disintegrated matter.

While the another matter emits as.

Achieving this stage will be the biggest success, as this stage has infinite union property (Figure 30).

The expansion governed with the law of gravitation which is

$$
F=G \cdot N / r^{2}, \quad N=M^{2}
$$

After this the capture field quanta moves according to

$$
(N+M)^{2} \cdot n=2 \cdot n \cdot N /(-M)
$$

Now this expanding Figure 31 it expands horizontally and vertically.

This discontinuity of horizontal method increases till the point where vertical expansion of structure achieves $0.9770847203 M=0$ condition which is entering rule diff. between ramanujan entering and dark matter Entering.

Future Scope-This law has scope in the following fields:

1) Laser and photonics.

2) Expansion of universe.

3) Connecting with other Galaxies.

4) Unlimited power sources.

5) Intense penetrating power.

6) Use in the medical field such radio therapy.

7) Use as weapons.

8) Use in diamond, mining, space and vending industry.

9) Research in almost every field of physics and chemistry.

10) Always something new to find.

\section{Cosmic Form of Nishant Effect}

In Figure 32, Figure 33, as known according to non-interactive mechanics and gtr that case of euclidean geometry turns into riemannian geometry, when mass-particle director value of $\frac{1}{4} \sqrt{\frac{8 m^{3} \pi}{\beta^{3}}} \cdot e^{\frac{\beta p^{2}}{2 m}}$ (mentioned in non-interactive mechanics) each works on the straight line and bend it in a curved line. So for the cosmic form of nishant effect is mentioned above. As the centered $n$ is matter emitter as $n=-\frac{4 \pi V}{3 h^{3}} \cdot \frac{1}{4} \sqrt{\frac{8 m^{3} \pi}{\beta^{3}}} \cdot e^{\frac{\beta p^{2}}{2 m}}$. 


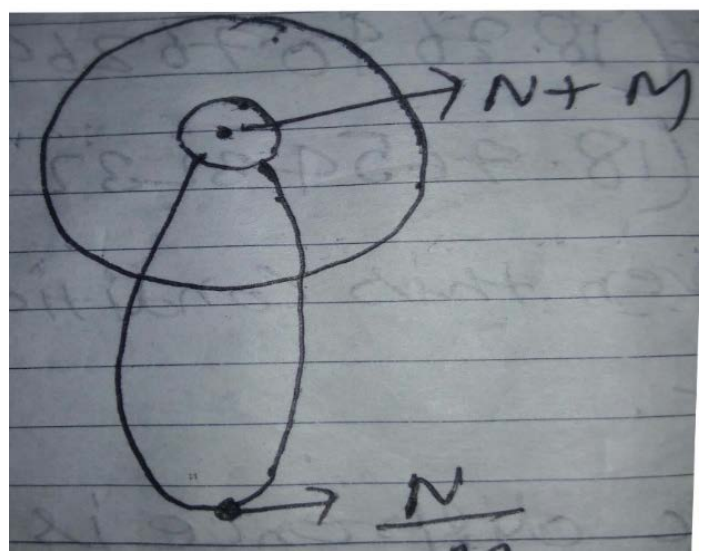

Figure 30. Expansion of class zero particle in horizontal and vertically.

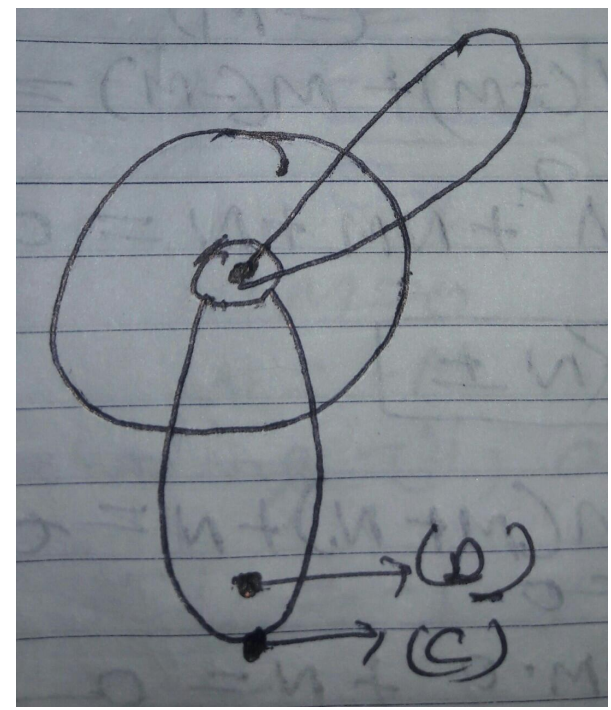

Figure 31. Extension of class zero particle in different dimensions.

\section{$E^{n}=(2(h V m a x-h V o)) / v^{2}$}

$\left(g i e^{\alpha+\beta E i}-e^{-\alpha-\beta E i}\right)^{2 . n}$

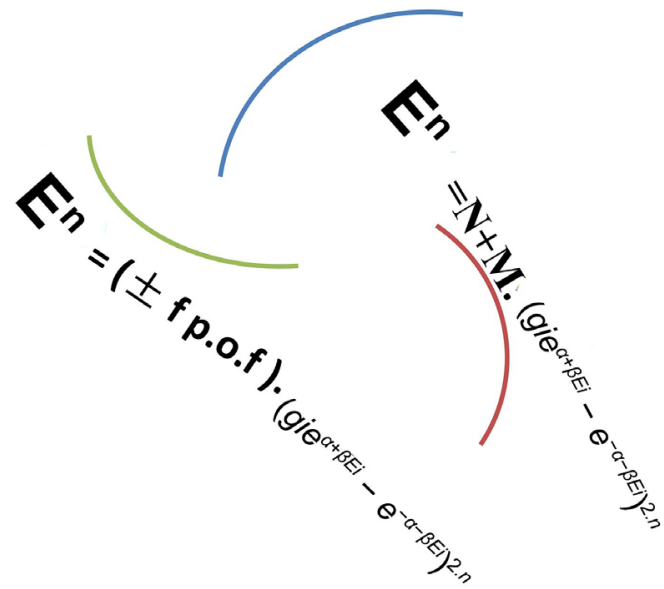

Figure 32. Figure of Nishant effect at lab. 


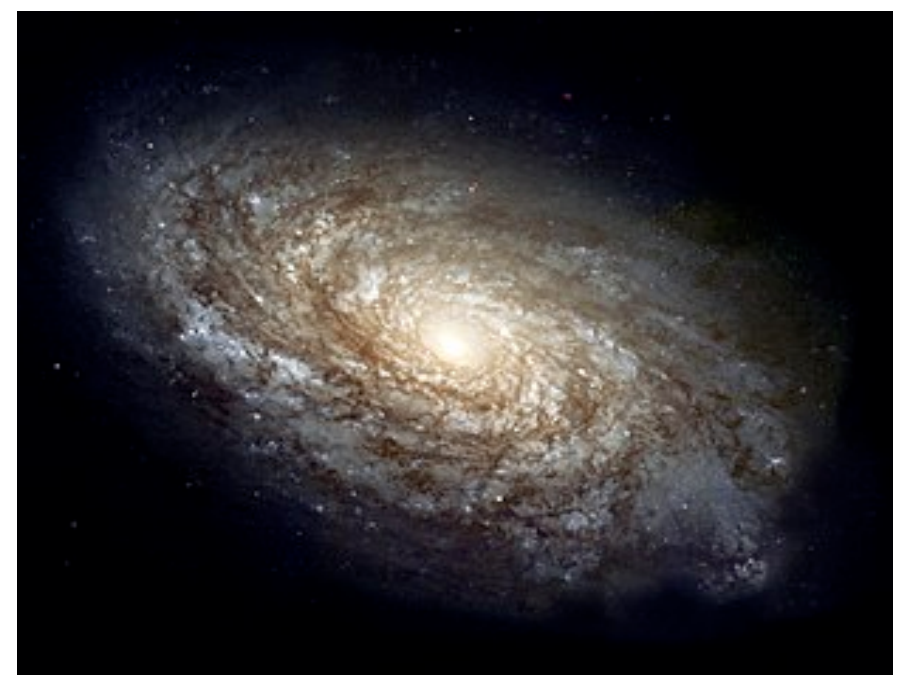

\section{https://en.wikipedia.org/wiki/Galaxy}

Figure 33. Nishant effect at cosmic level.

In first figure the total energy of all boundaries is $8 e$. And one longitudinal line we have the energy of $e^{n}=\frac{2(h v m a x-h v o)}{v^{2}} \cdot\left(g i e^{\alpha+\beta E i}-e^{-\alpha-\beta E i}\right)^{2 \cdot n}$ for $c=\left(g i e^{\alpha+\beta E i}-e^{-\alpha-\beta E i}\right)$ and $h V \max \gg h V 0$.

We have $e^{n}=\frac{2(\text { hvmax })}{v^{2}} \cdot(C)^{2 \cdot n}$ for $n=1$ and $\frac{(C)^{2}}{(v)^{2}}=1 \cdot e=2$ emax $=1$ due to this total energy of first figure system is $8 e+2 e=10 e$.

But if we consider central black joint part as pure matter from the equation of nishant effect

$$
E^{n}=\frac{2(h v m a x-h v o)}{v^{2}} \cdot\left(g i e^{\alpha+\beta E i}-e^{-\alpha-\beta E i}\right)^{2 \cdot n}
$$

The case when non-interactive particle is not present in the matter field than the cell will be empty, so $g i=0$, so above equation turns as

$$
E^{n}=\left(-e^{-\alpha-\beta E i}\right)^{2 \cdot n} E^{n}=\frac{2(h v m a x-h v o)}{v^{2}} \cdot\left(-e^{-\alpha-\beta E i}\right)^{2 \cdot n}
$$

In this condition hvmax becomes very low as no non-interactive particle is present in the field to hold the matter so $h w 0$ becomes $h v 0 \gg h v m a x$ for this above equation turn as

$$
\begin{gathered}
E^{n}=\frac{2(-h V o)}{v^{2}} \cdot\left(-e^{-\alpha-\beta E i}\right)^{2 \cdot n} \\
E^{n}=\frac{2(h V o)}{v^{2}} \cdot\left(e^{-\alpha-\beta E i}\right)^{2 \cdot n}
\end{gathered}
$$

For $e^{-\alpha}=$ constant above equation is 


$$
E^{n}=\frac{2(h V o)}{v^{2}} \cdot\left(e^{-\beta E i}\right)^{2 \cdot n}
$$

For $n=1$

$$
E=\frac{2(h V o)}{v^{2}} \cdot\left(1-\frac{2 h v}{k T}\right)
$$

Taking only first term we get

$$
E=\frac{2(h V o)}{v^{2}}
$$

For $V 0=V=\frac{C}{\lambda}$

$$
E=\frac{2(h c)}{v^{2} \lambda}
$$

For $\lambda=\frac{h}{m V}$

$$
\begin{gathered}
E=\frac{2(h c) m v}{v^{2} h} \\
E=\frac{2 m c}{V}
\end{gathered}
$$

Now for the condition where $v$ is just or approximately equals to $c$ we have $v=c$ this gives

$$
\begin{gathered}
E=\frac{2 m V}{V} \\
E=2 m
\end{gathered}
$$

For $n$ number case above equation is

$$
e=2 n \cdot m
$$

For $n=\frac{1}{2}$

$$
\begin{gathered}
E=2 \cdot \frac{1}{2} \cdot M \\
E=M
\end{gathered}
$$

Then the total energy is

$$
E \text { net }=10 E+E=11 E
$$

\section{Another Cosmological Aspect of Universe Stability also Our Earth Stability}

In my paper I describe about the infinite river of class zero particles with equa-

$$
\text { tion }\left(\frac{N}{-\frac{4 \pi V}{3 h^{3}} \cdot \frac{1}{4} \sqrt{\frac{8 m^{3} \pi}{\beta^{3}}} \cdot e^{\frac{\beta p^{2}}{2 m}}}\right)^{2 n}=2 \frac{N}{-\frac{4 \pi V}{3 h^{3}} \cdot \frac{1}{4} \sqrt{\frac{8 m^{3} \pi}{\beta^{3}}} \cdot e^{\frac{\beta p^{2}}{2 m}}} .
$$


On the L.H.S of equation shows river of class zero, and the edges of all theories which shows the edge of universe or every grand unified theories at the edges of these theories this river of class zero particle is present always. Now extending in the (ref. No. 10) theories there is the equation of the large scale structure of the universe is

$$
\left(\frac{N}{-M}\right)^{4 n-2}=4 n^{2}\left(\frac{N}{-M}\right)^{1+\left(\frac{N}{-M}\right)-1}
$$

For $n=1 / 2$ equation is valid. Now taking L.H.S of equation can be as that as four class zero particles moving linear direction and two particles of another class zero wave moving in opposite direction of 4 class zero particle now as according to Equation (2) reverse class zero particles will strike with 4 inversely and axially then the figures can be scened as.

First the opposite wave two particles unites then these conditions arise in high energy physics experiments a pyramidal shape observe the size and class of pyramid depend on the energy provide to the source, then it comes to the rest, this new state is Klein-Gordon relativistic quantum mechanical system and again the resulted structure putting to the high energy it covert into curved geometry which this follows the Riemannian geometry which behaves as same as General Relativity theory. As the temperature slowing by the help of mechanical instruments this curved rod turns into linear rod which follows Euclidean geometry.

1) So the new particle or COSMIC WATER can be achieved by this process.

2) We can expand our universe, splanets and human body by this experiment.

3) At last it is useful to expand a body mortal and Non-mortal objects (Figure $34)$.

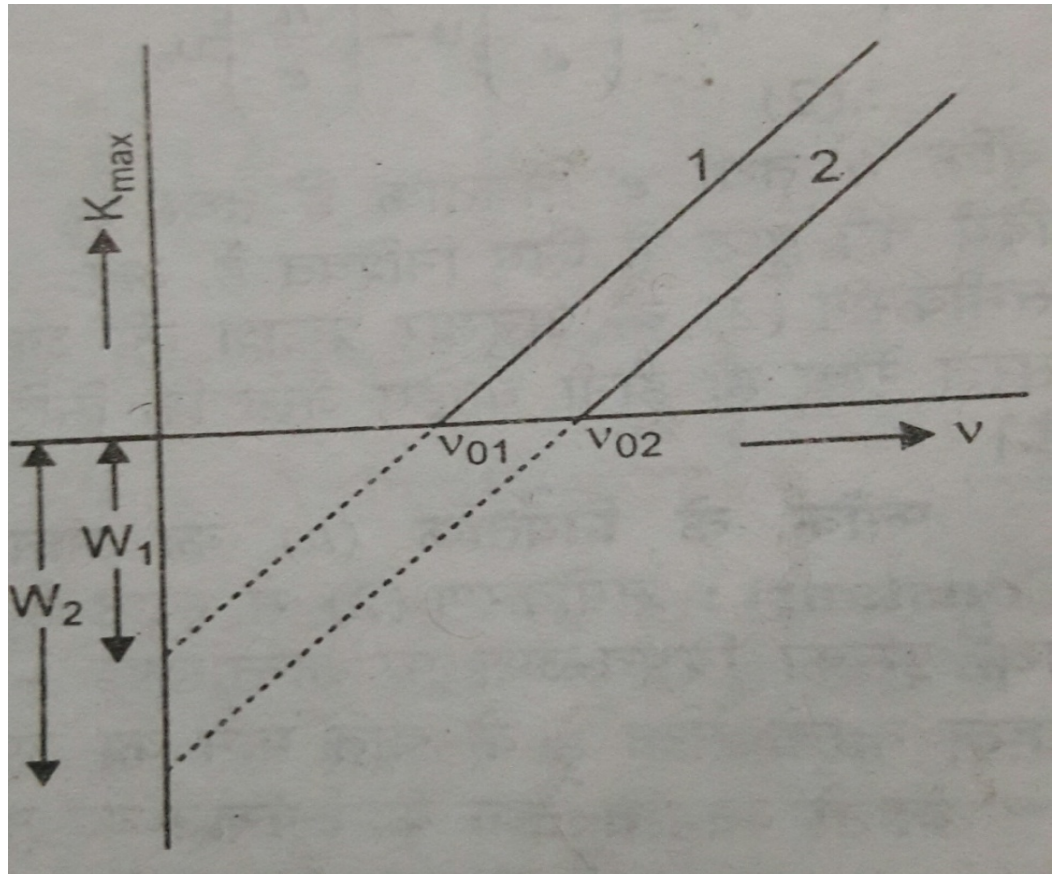

Figure 34. relation of positive energy axes with negative energy axes. 


\section{Discussion}

In near future whenever the quantum mechanical system forms in the space we have the system to find out the expansion rate of universe. We have linear and dirachamiltonian to understand the expansion form of linear and diagonal behaviour for the expansion of relativistic quantum mechanical system. We have new right angle triangle theorem to understand the geometrical expansion process for the expansion of astronomical objects. Then we have five factors to expansion of universe.

The modified black body radiation is based on non interactive mechanics and $\mathrm{m}$. Plank quantum hypothesis and the frequency and wavelength laws of a body without plank constant. Then four possibility of plank constant. Velocity of light, black holes and non intractable particle with respect to each other. Nature of non intractable particles, zeroes of fields and 1's in universe in other words reaction types. Scopes of the expansion of universe, cosmic form of the universe. Energy of $11 e$ around relativistic quantum mechanical system.

\section{Conclusion}

For the movement of elementary particle in the various theories of quantum mechanics and in various electronic system, a relativistic quantum mechanics system exists, which works in cross form, bound with the rules of relativistic quantum, classical mechanics. This shows that linear hamiltonian is stable at quantum mechanical level. This cross quantum mechanical system has a perfect black body at the centre where all four states combined this perfect black body make this system stable. Then we studied the $1 / 4$ th part of the system in form of right triangle theorem according to that "linearly the value of hypotenuse 'cd' of a right triangle $b c d$ is equal to the perpendicular line $a b$, and the system $b c+b d$, $\mathrm{dc}$ and $\mathrm{ab}$ are equal to each other". Then we observed that how this system works at an astrophysical level, in cosmic form of nishant effect which is same as milky way galaxy. Then we studied that when all the four parts are following the right angle theorem, the quantum mechanical system for the movement of elementary particles is surrounded with the energy of $10 e+e$.

\section{Funding Sources}

This research did not receive any specific grant from funding agencies in public, commercial, or not-for-profit sectors.

\section{Conflicts of Interest}

The author declares no conflicts of interest regarding the publication of this paper.

\section{References}

[1] Satyaprakash (2015) Advanced Quantum Mechanics, KNRN. 
[2] Sharma, N.K. (2020) On the Development of Photo-Electric Effect. International Journal of Scientific Research in Physics and Applied Sciences, 8, 20-23. https://doi.org/10.26438/ijsrpas/v8i1.2023

[3] Sharma, N.K. (2020) Cosmological Concepts Astrophysical Extension of Non Intracative Mechanics. International Journal of Scientific Research in Physics and Applied Sciences, 8, 27-46. https://doi.org/10.26438/ijsrpas/v8i1.2746

[4] Sharma, N.K. (2019) Non-Interactive Mechanics. Journal of Nuclear and Particle Physics, 9, 18-41.

[5] Satyaprakash (2015) Statistical Mechanics, KNRN.

[6] Sharma, N.K. (2020) Dark Energy: Particle and Astrophysical Examination. International Journal of Scientific Research in Physics and Applied Sciences, 8, 47-58. https://doi.org/10.26438/ijsrpas/v8i1.4758 